\title{
The GeoSteiner Software Package for Computing Steiner Trees in the Plane: An Updated Computational Study
}

\author{
Daniel Juhl ${ }^{1}$, David M. Warme ${ }^{2}$, Pawel Winter ${ }^{3}$, and Martin Zachariasen ${ }^{4}$ \\ 1 Department of Computer Science, University of Copenhagen, DK-2100 \\ Copenhagen $\varnothing$, Denmark, juhl.daniel@gmail.com \\ 2 Group W, Inc., 8315 Lee Highway, Suite 400, Fairfax, Virginia, USA, \\ david@warme.net \\ 3 Department of Computer Science, University of Copenhagen, DK-2100 \\ Copenhagen $\varnothing$, Denmark, pawel@di.ku.dk \\ ${ }^{4}$ Department of Computer Science, University of Copenhagen, DK-2100 \\ Copenhagen $\varnothing$, Denmark, martinz@di.ku.dk
}

\begin{abstract}
The GeoSteiner software package has for more than 10 years been the fastest (publicly available) program for computing exact solutions to Steiner tree problems in the plane. The computational study by Warme, Winter and Zachariasen, published in 2000, documented the performance of the GeoSteiner approach - allowing the exact solution of Steiner tree problems with more than a thousand terminals. Since then, a number of algorithmic enhancements have improved the performance of the software package significantly. In this computational study we run the current code on the largest problem instances from the 2000-study, and on a number of larger problem instances. The computational study is performed using the commercial GeoSteiner 4.0 code base, and the performance is compared to the publicly available GeoSteiner 3.1 code base as well as the code base from the 2000-study.
\end{abstract}

Keywords: Euclidean Steiner tree problem, rectilinear Steiner tree problem, fixed orientation Steiner tree problem, exact algorithm, computational study.

\section{Introduction}

The Steiner tree problem in the plane asks for a shortest possible interconnection of a set of points under some given metric. The Euclidean and rectilinear Steiner tree problems in the plane are by far the most studied geometric Steiner tree problem variants $[11,12,14]$. Recently, the uniform and fixed orientation metrics have also received some attention due to applications in the physical design of integrated circuits $[4,5,25]$. A uniform orientation metric is given by a set of $\lambda \geq 2$ uniformly distributed orientations in the plane, and the goal is to compute a shortest possible interconnections where all line segments have one of the given orientations. The rectilinear metric is a uniform orientation metric with two legal 
orientations, namely the horizontal and vertical orientations. The Steiner tree problem in the plane is NP-hard for all interesting metrics $[6,9,10]$. For a comprehensive introduction to these problems, see the upcoming book by Brazil and Zachariasen [7].

Background and Motivation. Since the publication of the computational study by Warme, Winter and Zachariasen [24] in 2000, the GeoSteiner software package - released in 2001 - has been the fastest (publicly available) program for computing exact solutions to Steiner tree problems in the plane. The GeoSteiner approach has successfully been extended to uniform orientation metrics [19], to Euclidean and rectilinear problems with obstacles [13, 30], and to rectilinear group interconnection problems [28].

The computational effectiveness of the GeoSteiner approach is largely due to the fact that a minimum Steiner tree in the plane can be decomposed into small so-called full Steiner trees (FSTs); these are subtrees where Steiner points are interior vertices and terminals are leaves [24]. The GeoSteiner algorithm has two phases: FST generation and FST concatenation. In the first phase, all FSTs that can possibly appear in a minimum Steiner tree are enumerated. In the second phase, a subset of the generated FSTs is chosen such that they form a minimum Steiner tree. The first phase is obviously very metric dependent, while the second phase is purely combinatorial (and metric independent).

Since the publication of the 2000-paper [24], a number of (unpublished) algorithmic enhancements have improved the performance of the software package significantly. Some of these enhancements were part of the 2001 public release, while other have been added in later versions. These algorithmic engineering efforts cover the use of faster data structures, new heuristics for better pruning of FSTs, and better cutting methods in the branch-and-cut concatenation algorithm. The motivation of the current paper is to describe these improvements and document their effects, both for the publicly available GeoSteiner 3.1 code base and the commercial GeoSteiner 4.0 code base.

Our Contribution. We present an updated computational study on Steiner tree problems in the plane. Also, we document the main algorithmic enhancements made to the GeoSteiner software package. In the updated computational study we run the current code on the largest problem instances from the 2000study, and on a number of even larger problem instances. The computational study is performed using the commercial GeoSteiner 4.0 code base, and the performance is compared to the publicly available GeoSteiner 3.1 code base as well as the code base from the 2000-study.

As a general rule, the FST generation phase has a more predictive running time than the FST concatenation phase. As a consequence, the FST concatenation phase is usually the bottleneck when solving large-scale problem instances. The FST concatenation problem can either be solved as a minimum spanning tree problem in a hypergraph (as in the 2000-paper), or as a Steiner tree problem in an ordinary graph. The latter approach was studied and experimentally evaluated by Polzin and Vahdati Daneshmand [20] in 2003, and it appeared to have superior performance when compared the FST concatenation code of 
GeoSteiner 3.1. However, the software used in [20] is not publicly available; in fact, no software for solving the Steiner tree problem in graphs is - to the best of our knowledge - publicly available, so we have restricted our experiments to solving the FST concatenation problem as a minimum spanning tree problem in a hypergraph. However, we are planning to make the output from the FST generation available as instances of the Steiner tree problem in graphs, so other researchers can solve the FST concatenation problems using their own code.

Organisation of the Paper. In Section 2 we give a brief introduction to the GeoSteiner approach. A presentation of the major algorithmic enhancements made since the publication of the 2000-paper are given in Section 3. Computational results are presented in Section 4, and concluding remarks are given in Section 5 .

\section{GeoSteiner Approach}

Let $N$ be a finite set of points, or so-called terminals, in the plane, and assume that some metric is given. A naive algorithm for computing a minimum Steiner tree for $N$ under the given metric is to enumerate all full Steiner topologies for every subset of terminals, and then compute an FST for the given topology, or to decide that no such tree exists $[7,15]$. A minimum Steiner tree is then obtained by identifying a subset among the constructed FSTs that interconnects $N$ and has minimum length. However, since the number of terminal subsets is exponential in the size of $N$ - and the number of full Steiner topologies for each subset is super-exponential — this algorithm would show very bad scaling.

GeoSteiner follows the same two-phase approach as the naive algorithm, but reduces the work significantly by implicit instead of explicit enumeration of FSTs (for all subsets and all full Steiner topologies). Groups of FSTs that do not fulfill necessary structural properties are eliminated early — and in most cases without direct geometric construction.

\section{$2.1 \quad$ FST Generation}

The task of the FST generation phase is to determine an as small as possible superset of FSTs of a minimum Steiner tree. Consider some FST $T$ spanning a subset of $k$ terminals in $N$, where $3 \leq k \leq|N|$. We can assume that the Steiner points in $T$ have degree 3 . Steiner points with degree 4 or more can only appear under certain metrics, and in these cases they can be assumed to appear in FSTs that are easily identified [5]. The FST $T$ therefore has $k$ terminals as leaf nodes (with degree 1) and $k-2$ Steiner points as interior nodes (with degree 3 ).

For the Euclidean metric the edges in $T$ - which interconnect terminals and/or Steiner points - are straight line segments, and the edges at a Steiner point in $T$ meet at $120^{\circ}$ angles. For the rectilinear metric, and more generally for any fixed orientation metric, the edges of $T$ can also be assumed to be straight

edges, except possibly one so-called bent edge $p q$, which interconnects nodes $p$ and $q$ using exactly two straight line segments that meet at a corner point [5]. 
FST generation in GeoSteiner is performed by enumerating so-called branch trees. Consider FST $T$. If $T$ has a bent edge $p q$, then let $c$ be the corner point of $p q$; otherwise let $c$ the midpoint of any (straight) edge $p q$ in $T$. Imagine that we cut edge $p q$ at point $c$. We obtain two branch trees: $B_{1}$ rooted at $p$ having a stem (or ray) leaving $p$ along $p c$, and $B_{2}$ rooted at $q$ having a stem leaving $q$ along $q c$ (Figure 1). Note that all edges in a branch tree are straight edges.

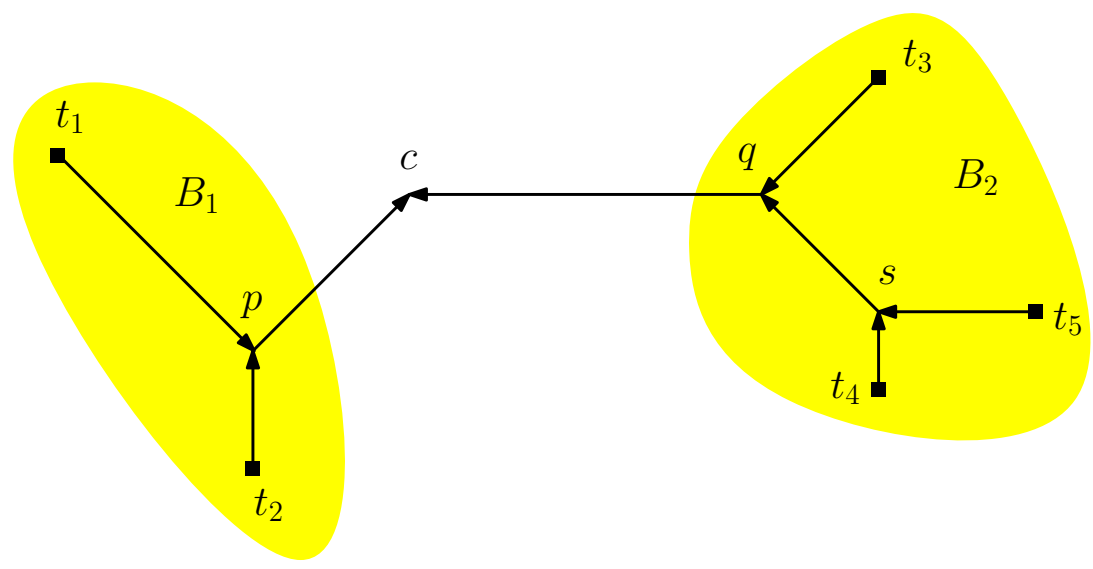

Fig. 1. An FST $T$ spanning five terminals $t_{1}, \ldots, t_{5}$ with a bent edge $p q . T$ can be obtained by joining two branch trees $B_{1}$ and $B_{2}$ with roots $p$ and $q$, respectively. The arrows indicate the paths from the terminals to the root of each branch tree. Branch tree $B_{1}$ is obtained by joining branch trees for terminals $t_{1}$ and $t_{2}$. Branch tree $B_{2}$ is obtained by joining a branch tree for terminal $t_{3}$ with a branch tree having root $s$ that spans terminals $t_{4}$ and $t_{5}$. The example is given for a fixed orientation metric.

Intuitively, we can think of a branch tree as an FST spanning a set of terminals and a single point at infinity. We define the size of a branch tree to be the number of terminals spanned by the branch tree. A branch tree of size 1 consists of a single terminal with a stem; such a branch tree has no Steiner points and the terminal is the root of the branch tree. A branch tree of size $k \geq 2$ has $k$ terminals and $k-1$ Steiner points, and can naturally be represented as a binary tree. Branch trees for the Euclidean problem are represented by equilateral points, and the root of a branch tree can be located anywhere on a so-called Steiner arc [26].

The generation phase of GeoSteiner enumerates branch trees of increasing size - essentially using a dynamic programming approach. Branch trees of size $k$ are obtained by joining branch trees of size $l$ with branch trees of size $k-l$, where $l$ runs from 1 to $\lfloor k / 2\rfloor$. An FST is obtained by identifying two branch trees where the stems can be made to overlap in opposite directions (for the Euclidean metric) or intersect at an appropriate angle (for fixed orientation metrics). 
Branch trees and FSTs are eliminated from consideration by performing a number of pruning tests. These tests are metric dependent, but are based on similar structural properties such as empty regions and upper bounds on edge lengths in a FST [19, 26, 27]. A simple upper bound on the length of any edge in a minimum Steiner tree for $N$ is to take the longest edge in a minimum spanning tree for $N$ [26]; in the following we let $b_{\max }$ denote this upper bound for a given set of terminals $N$.

\section{$2.2 \quad$ FST Concatenation}

The FST concatenation problem can be modelled as an instance of the minimum spanning tree in hypergraph (MSTHG) problem: given a hypergraph $\mathbf{H}=(V, \mathbf{E})$, and a weight function $w: \mathbf{E} \mapsto \mathbb{R}$, find a subset $\mathbf{T} \subseteq \mathbf{E}$ such that $\mathbf{T}$ is a spanning tree of $\mathbf{H}$ that minimizes $w(\mathbf{T})=\sum_{\mathbf{e} \in \mathbf{T}} w(\mathbf{e})$. FST concatenation instantiates $V$ as the set of terminals $N, \mathbf{E}$ as the set of all FSTs (each FST is a hyperedge connecting 2 or more vertices in the hypergraph), and $w(\mathbf{e})$ as the geometric length of FST e using the appropriate distance metric. Note that even deciding the existence (or not) of a spanning tree within a general hypergraph is NP-complete [23], so FST concatenation is in general an NP-hard optimization problem.

The MSTHG problem is solved using the following integer program (IP):

$$
\begin{aligned}
& \text { Minimize } \sum_{\mathbf{e} \in E} c_{\mathbf{e}} x_{\mathbf{e}} \\
& \text { Subject to } \\
& \sum_{\mathbf{e} \in \mathbf{E}}(|\mathbf{e}|-1) x_{\mathbf{e}}=|V|-1, \\
& \sum_{\mathbf{e} \in \mathbf{E}} \max (|\mathbf{e} \cap S|-1,0) x_{\mathbf{e}} \leq|S|-1, \quad \forall S \subset V,|S| \geq 2 \\
& 0 \leq x_{\mathbf{e}} \leq 1, \quad \forall \mathbf{e} \in \mathbf{E} \\
& x_{\mathbf{e}} \in \mathbb{Z}, \quad \forall \mathbf{e} \in \mathbf{E}
\end{aligned}
$$

where $c \in \mathbb{R}^{|\mathbf{E}|}$ is the cost vector such that $c_{\mathbf{e}}=w(\mathbf{e})$ for all $\mathbf{e} \in \mathbf{E}$, and $x \in \mathbb{R}^{|\mathbf{E}|}$ is the solution vector. The following two theorems were proved by Warme [23]:

Theorem 1. Let $\mathbf{T} \subseteq \mathbf{E}$ be any spanning tree of $\mathbf{H}=(V, \mathbf{E})$. Let $\tilde{x} \in \mathbb{R}^{|\mathbf{E}|}$ such that $\tilde{x}_{\mathbf{e}}=1$ if $\mathbf{e} \in \mathbf{T}$ and $\tilde{x}_{\mathbf{e}}=0$ otherwise ( $\tilde{x}$ is the incidence vector of $\mathbf{T}$ ). Then $\tilde{x}$ is an integer feasible solution to IP.

Theorem 2. Let $\tilde{x}$ be any feasible integer solution to IP. Let $\mathbf{T}=\{\mathbf{e} \in \mathbf{E}$ : $\left.x_{\mathbf{e}}=1\right\}$, so that $\tilde{x}$ is the incidence vector of $\mathbf{T}$. Then $\mathbf{T}$ is a spanning tree of $\mathbf{H}$.

The LP relaxation of IP is obtained by removing constraint (4). This relaxation produces very tight lower bounds in practice. Integrality is recovered via branch-and-bound upon fractional variables of the LP relaxation. Note that there are exponentially many constraints (2). These constraints are added to the 
formulation dynamically as violations are discovered. This separation problem can be solved in polynomial time by reduction to flows using the techniques described in [23].

\section{Algorithmic Enhancements}

The 2000-paper [24] combined the Euclidean FST generation algorithm from [26], the rectilinear FST generation algorithm from [27], and the branch-and-cut based FST concatenation algorithm described in [23]. All generated FSTs were considered in the branch-and-cut algorithm. No FST pruning (or hypergraph reduction) was used, and the algorithms were not integrated at that time.

The GeoSteiner implementation has improved considerably since the publication of [24], rendering those results obsolete. The following table summarizes the major versions of GeoSteiner, their release dates, and major feature improvements, and the section numbers refer to sections below that describe these features in more detail. GeoSteiner 4.0 is currently a proprietary product (not open source)

\begin{tabular}{|l|l|l|l|}
\hline Version & Date & Major New Features and Improvements & Section \\
\hline$[24]$ & $\begin{array}{l}\text { March } \\
1998\end{array}$ & & \\
\hline 3.0 & January & New implementation of FST generators & 3.1 \\
& 1999 & $\begin{array}{l}\text { No compiled-in limits on problem size } \\
\text { Save/restore LP basis when switching nodes }\end{array}$ & \\
& & Handle abstract hypergraph problems & \\
\hline 3.1 & February & New greedy Euclidean heuristic & 3.2 \\
& 2001 & Numeric stability improvements & 3.3 \\
& & FST pruning added & 3.4 \\
& & Improved variable fixing in branch-and-cut & 3.5 \\
& Improved strong branching heuristics & 3.6 \\
\hline 4.0 & February & Callable library & 3.7 \\
& 2006 & New bottleneck Steiner distance data structure & 3.8 \\
& & Uniform orientations FST generator & 3.9 \\
& & Cut generation improvements & 3.10 \\
& & Local cuts & 3.11 \\
\hline
\end{tabular}

\subsection{New FST Generator Implementations}

The original Euclidean and rectilinear FST generators were implemented in C++ using LEDA $[17,18]$. These were replaced with new highly tuned implementations written in C. Both of the new FST generators replaced the LEDA and other data structures with more efficient, low-level data structures that are customized to provide only the necessary operations, and are much more friendly to modern cache hierarchies and deeply pipelined CPU architectures. They also use a much better hash table and linear time check for duplicate FSTs (the shortest is retained). 
Improvements to the Euclidean FST Generator. The new Euclidean FST generator uses a range search idea originally suggested by Althaus [1] to identify relevant pairwise branch trees as follows. Let $b_{\max }$ be the length of the longest edge in a minimum spanning tree for $N$ - which is the same as the maximum bottleneck Steiner distance (see Section 3.8). Then no edge in any minimum Steiner tree for $N$ can be longer than $b_{\max }$. Consider two branch trees $B_{1}$ and $B_{2}$. Let $\gamma$ be the distance between the roots of $B_{1}$ and $B_{2}$ (see Section 2.1); if $B_{1}$ and $B_{2}$ are terminals, $\gamma$ is simply the distance between these terminals. Clearly, if $\gamma>2 b_{\max }$ then no feasible combination of $B_{1}$ and $B_{2}$ exists, since at least one of the new edges would have a length greater than $b_{\max }$. Thus, given a branch tree $B_{1}$, we can use a range search data structure to identify all branch trees that are within distance $2 b_{\max }$ of $B_{1}$ (where distance is defined as above). A simple bucket structure was used as data structure: the minimum rectangle containing the set of terminals $N$ is divided into $K \times K$ subrectangles. One bucket structure was constructed for each size of branch trees, allowing fast identification of branch trees of appropriate size. This method significantly improves the running time for large problem instances, especially those with a uniform distribution of the terminals.

In addition, many uses of trigonometric functions were replaced with vector operations (e.g., dot and cross product), and more efficient data structures were implemented for representing the terminals spanned by branch trees (or equilateral points).

Improvements to the rectilinear FST Generator. The so-called empty rectangles matrix was reorganized as a lower-triangular bit matrix to reduce its size and improve locality (see [27] for details). In addition, several crucial lowlevel geometric primitives (macros) were replaced with new implementations that use no conditional branches. By reducing pipeline flushes, these macro changes produced about a 3 -fold additional speed improvement on top of all the other improvements.

\subsection{New Greedy Euclidean Steiner Tree Heuristic}

The Zachariasen-Winter greedy heuristic for Euclidean Steiner trees [29] was added as an alternative to the existing Smith-Lee-Liebman heuristic [22].

\subsection{Numerical Stability Improvements to Euclidean FST Generator}

Several rounds of changes were made to improve the numerical stability of the Euclidean FST generator. First, dynamically computed (relative) tolerances were used for all floating-point comparisons. Behavior for point sets located away from the origin was improved by translating the entire point set to a new origin computed to be both "central" to the point set and to have relatively few significant bits. Next, all computations for a single equilateral point were performed with 
respect to an origin located at one of its terminals, thereby increasing the number of significant bits available for these computations. All movement of Steiner arc endpoints are encapsulated into new routines that are much more careful to make sure that the arc is never shortened any more than can be justified numerically.

Finally, the code was modified to use the GNU multi-precision arithmetic package (GMP) under control of a Level switch, which defaults to level 0. At Levels 1 and 2, GMP exact rational arithmetic is used to compute: (1) the exact coordinates (in $\mathbb{Q}(\sqrt{3})$ ) of each equilateral point; (2) the closest double-precision representation of each equilateral point's coordinates; and (3) the closest doubleprecision representation of the length of each FST (in $\sqrt{\mathbb{Q}(\sqrt{3})}$ ). Conversion of $\mathbb{Q}(\sqrt{3})$ into numeric form is accomplished using a Newton iteration, with initial approximation computed via floating-point. At Level 1, a single high-precision Newton iteration is used to refine this value before converting to the nearest double-precision representation. At Level 2, Newton iterations are continued until a formal convergence test has verified that the value is correct to at least $1 / 2$ ULP of double-precision. These computations are performed only on equilateral points and FSTs that have passed all screening tests, as a part of storing them in their final form. This prevents floating-point errors from accumulating as equilateral points are recursively constructed from smaller equilateral points. This in turn permits more conventional relative numeric tolerancing techniques to be used in a sound manner in the more intensively traversed portions of the code. On large random instances, GMP Level 2 adds only about $1-2 \%$ to the run time, but significantly improves the numerical accuracy and stability of the generated FSTs.

\subsection{New FST Pruning}

The FST pruning algorithm of Fößmeier and Kaufmann [8] was implemented for all metrics. (The previous pruning codes were slow, relatively ineffective, and completely specific to either the Euclidean or rectilinear metric $[21,26]$.) The idea of the Fößmeier and Kaufmann pruning algorithm is to test whether an FST $F$ must be part of a larger full component in a minimum Steiner tree. More specifically, the pruning algorithm searches for an FST $F$ and a terminal $t$ with the following properties:

1. Terminal $t$ is not spanned by $F$, and has (minimum) distance $\gamma$ to some edge $(p, q)$ in $F$.

2. The longest edge on every path $P$ from $t$ to $p$, or from $t$ to $q$, is longer than $\gamma$ (where the edges of $P$ come from the set of generated FSTs).

Assuming that $F$ appears in a minimum Steiner tree leads to a contradiction, since we can add a connection of length $\gamma$ from $t$ to $(p, q)$, and remove an edge of length more than $\gamma$ on the cycle that is created. Therefore a shorter tree can be constructed, and $F$ can be eliminated from consideration. 
Efficient implementation of this pruning algorithm requires significant care, but the method is very powerful in practice and typically removes at least half of the generated FSTs. Here we give some of the most important implementation details:

1. First the so-called pruning graph $G_{P}=\left(V_{P}, E_{P}\right)$ is computed. The vertex set $V_{P}$ consists of the terminals $N$ and the Steiner points in all generated FSTs. The edge set $E_{P}$ consists of the edges of all generated FSTs. The edge set is sorted by non-decreasing length (under the given metric), and necessary information is attached to the edge set such that a sorted list of edges efficiently can be generated for any given subset of FSTs.

2. For each FST $T$ the list of compatible FSTs is generated. These are FSTs that can appear together with $T$ in some minimum Steiner tree. Consider some FST $T^{\prime} \neq T$. If $T^{\prime}$ has two or more terminals in common with $T$, then $T^{\prime}$ is not compatible with $T$. Consider the case where $T^{\prime}$ has a single terminal in common with $T$. If a shorter tree interconnecting the terminals spanned by $T$ and $T^{\prime}$ can be constructed, then $T^{\prime}$ is not compatible with $T$; first simple heuristics and finally an exact algorithm based on the FSTs already generated is used for constructing a tree interconnecting the terminals spanned by $T$ and $T^{\prime}$.

3. For each FST $T$ the set of terminals that are within distance $b_{\max }$ to $T$ are identified (see Section 2.1). The distance from a terminal $t$ to an edge $(p, q)$ in $T$ is computed as the difference in length between a minimum Steiner tree for $t, p$ and $q$, and the length of edge $(p, q)$, that is, the increase in length that is needed to insert $t$ on edge $(p, q)$. The list of terminals is sorted by their minimum distance to $T$ (shortest distance first).

4. When attempting to prune an FST $T$, the sorted list of close terminals is traversed. Consider a terminal $t$ having distance $\gamma$ to some edge $(p, q)$ in $T$. A (forest) graph $G_{T}=\left(V_{T}, E_{T}\right)$ with vertex set $V_{T}=V_{P}$ is constructed; the edge set $E_{T}$ is formed by using Kruskal's minimum spanning tree algorithm on the sorted edge set $E_{P}$ - but only including edges from FSTs that are compatible with $T$. As soon as an edge of length $\gamma$ or more is reached, Kruskal's algorithm stops. Now, if $t$ and $p$, as well as $t$ and $q$, are in different connected components in $G_{T}$, then $T$ can be pruned. Note that the graph $G_{T}$ has to be built only once for the sorted list of close terminals to $T$.

Clearly, as FSTs are pruned, the list of compatible FSTs for each remaining FST must be recomputed. This is done at appropriate intervals in order to save running time.

In addition to pruning FSTs, FSTs that are required to be part of any minimum Steiner tree can be identified. If a terminal $t$ only is spanned by a single remaining FST $T$, then $T$ must be part of any minimum Steiner tree. More generally, if a connected component of required FSTs has a single adjacent FST, then this FST is required. Finally, any FST that is not compatible with a required FST can be pruned. 


\subsection{Improved Variable Fixing in Branch-and-Cut}

Variable fixing in the branch-and-cut algorithm for solving the FST concatenation problem was improved (see Section 2.2). Let $Z$ be the current optimal LP objective value for a node, $d_{\mathbf{e}}$ be the reduced costs for edge e, and $U$ be the best upper-bound available. Previous variable fixing was activated when a non-basic edge variable $x_{\mathbf{e}}$ satisfied $Z+\left|d_{\mathbf{e}}\right|>U+\epsilon$, with small $\epsilon>0$. The new implementation maintains new vectors $Z_{l b 0}$ and $Z_{l b 1}$. Element e of these vectors stores the highest value of $Z+\left|d_{\mathbf{e}}\right|$ ever seen while $x_{\mathbf{e}}$ is non-basic at 0 (or 1), respectively, and represent lower bounds on the objective value obtained by forcing $x_{\mathbf{e}}=0$ (or $x_{\mathbf{e}}=1$ ), respectively. This allows variables to be fixed even when the instantaneous value of $Z+\left|d_{\mathbf{e}}\right|$ is not sufficiently high to produce a cutoff. The quantities $Z_{l b 0}$ and $Z_{l b 1}$ are also used in the branch variable selection methods, as discussed below.

\subsection{Improved Strong Branching Heuristics}

A new heuristic ranking of branch variable candidates in the branch-and-cut algorithm for the FST concatenation problem was introduced. Each node maintains a vector bheur. Element bheur $r_{\mathbf{e}}$ indicates how much the corresponding value of $x_{\mathbf{e}}$ has been changing in recent iterations. We set bheur $=0$ initially. Let $x$ be the current LP solution, $x^{\prime}$ be the previous LP solution, and bheur ${ }^{\prime}$ be the previous bheur. Then

$$
\text { bheur }=0.75 \text { bheur }^{\prime}+\left|x-x^{\prime}\right| .
$$

For each fractional branch variable candidate $x_{j}$, we compute a closest rational approximation $N_{j} / D_{j}$, and then

$$
\operatorname{rank}_{j}=\operatorname{bheur}_{j}\left(\left(D_{j}-1\right)+\left|N_{j}-D_{j} / 2\right|\right) .
$$

Candidate branch variables are sorted into increasing order by $r_{a n k}$. Strong branch testing is performed in that order. This favors variables that have been "stuck" at "simple fractional values" (e.g., 1/2, 3/4, etc.) for several iterations. We test variables in this order until either: (1) one or more variables are fixed; (2) a cutoff is obtained; (3) all candidates are tested; or (4) when $K$ consecutive variables have been tested without finding an improving candidate, where $K=2\left\lfloor\log _{2}\right.$ (NumFrac) $\rfloor$, where NumFrac is the number of fractional branch variable candidates.

Finally, improvements were made to the interplay between the primal upper bound heuristic, variable fixing, and branch variable testing. The primal upper bound heuristic is applied to every LP solution obtained while testing branch variables. New upper bounds discovered in this way provide opportunities for additional variable fixing. Any branch variable candidate that gets fixed in this manner while testing the candidates causes branch variable selection to abort, allowing cut generation to resume at the current node in lieu of branching. 


\subsection{Callable Library}

In GeoSteiner 4.0, the code was completely restructured and re-factored to encapsulate the algorithms as a subroutine library, allowing GeoSteiner 4.0 to be much more easily used as a "black box" within other applications.

\subsection{New Bottleneck Steiner Distance Data Structure}

Consider a minimum spanning tree $\bar{T}$ for $N$. The bottleneck Steiner distance between two terminals $t_{1}, t_{2} \in N$ is the length of the longest edge on the path between $t_{1}$ and $t_{2}$ in $\bar{T}$. Bottleneck Steiner distances between every pair of terminals can trivially be determined in $O\left(n^{2}\right)$ time by doing a depth-first traversal in $\bar{T}$ from every terminal. This gives constant-time lookup of bottleneck Steiner distances, but requires $\Theta\left(n^{2}\right)$ space. For large problem instances, it is more efficient to use the data structure suggested in [16] which uses $\Theta(n)$ space; the preprocessing time is $O(n \log n)$, and queries can be made in $O(\log n)$ time. The FST generator was set to use this $\Theta(n)$ space data structure when the number of terminals was greater than 100 .

\subsection{FST Generator for Uniform Direction Metrics}

A new FST generator for uniform orientation metrics was added. A description of this algorithm appears in [19]. This FST generator was coded in C using similar techniques as the existing Euclidean and rectilinear FST generators.

\subsection{Improved Cut Generation}

Significant enhancements were made to the separation procedures in the branchand-cut algorithm that affect which constraints are (and are not) generated. These changes significantly decrease the number of optimize/separate iterations needed to converge to an optimal subtour relaxation, and also result in LP instances that are more sparse and that solve more quickly.

\subsection{Local Cuts}

The branch-and-cut algorithm was improved by adding local cuts — originally devised by Applegate et al. [2] for the traveling salesman problem. We adapted them for the spanning tree in hypergraph problem as follows. Let $\mathbf{H}=(V, \mathbf{E})$ be a hypergraph. Let $S T P(\mathbf{H})$ be the spanning tree in hypergraph $\mathbf{H}$ polytope (i.e., the convex hull of all incidence vectors corresponding to spanning trees of $\mathbf{H})$.

Let $\bar{x}$ be a fractional LP solution vector to separate from $S T P(\mathbf{H})$. Let $S \subset$ $V$, and consider the sub-hypergraph $\mathbf{H}^{\prime}=\left(S, \mathbf{E}^{\prime}\right)$ of $\mathbf{H}$ induced by $S$. Let $\mathbf{T} \subseteq \mathbf{E}$ be any spanning tree of $\mathbf{H}$, and consider its image in $\mathbf{H}^{\prime}$, which will always be a forest (possibly empty, i.e., no edges). Let $F P\left(\mathbf{H}^{\prime}\right)$ be the forest in hypergraph $\mathbf{H}^{\prime}$ polytope (i.e., the convex hull of all incidence vectors corresponding to forests 
in hypergraph $\mathbf{H}^{\prime}$ ). Let $\bar{x}^{\prime}$ be the image of $\bar{x}$ with respect to $\mathbf{H}^{\prime}$. We produce a constraint $\bar{y} x \leq 1$ that separates $\bar{x}^{\prime}$ from $F P\left(\mathbf{H}^{\prime}\right)$, or prove that none exists because $\bar{x}^{\prime} \in F P\left(\mathbf{H}^{\prime}\right)$.

We start with a set $F$ of forests in $\mathbf{H}^{\prime}$, represented as incidence vectors. (Initially, $F$ contains single-edge forests, one for each edge in $\mathbf{E}^{\prime}$.) Let $\bar{y}$ be the optimal solution to the following linear program:

$$
\text { Maximize } \quad Z(y)=\bar{x}^{\prime} y
$$

Subject to

$$
f y \leq 1 \quad \forall f \in F
$$

Let $\bar{y}$ be an optimal solution to this LP with objective $\bar{Z}$. If $\bar{Z} \leq 1$, then $\bar{x}^{\prime} \in \operatorname{conv}(F) \subset F P\left(\mathbf{H}^{\prime}\right)$, and we are done - no separating hyperplane exists. Otherwise $\bar{Z}>1$ and $\bar{y} x \leq 1$ separates $\bar{x}^{\prime}$ from $F$ - but this constraint may not be valid for $F P\left(\mathbf{H}^{\prime}\right)$. We test validity by solving the following maximumweight forest problem:

$$
\text { Maximize } \quad Q(f)=\bar{y} f
$$

Subject to

$$
\begin{array}{r}
f \in F P\left(\mathbf{H}^{\prime}\right) \\
f \in\{0,1\}^{\left|\mathbf{E}^{\prime}\right|}
\end{array}
$$

This is an NP-hard optimization problem that we easily reduce to the MST in hypergraph problem as follows. First produce a minimization problem by negating the edge weights. Construct a new hypergraph $\mathbf{H}^{\prime \prime}$ from $\mathbf{H}^{\prime}$ by adding 1 new vertex $t$ and a zero weight edge from $t$ to each $v \in S$. There is an obvious oneto-one correspondence between each spanning tree in $\mathbf{H}^{\prime \prime}$ and the corresponding forest in $\mathbf{H}^{\prime}$. Let $\bar{f}$ be any feasible integer solution to IP1 having the objective

$$
Q(\bar{f})=\bar{y} \bar{f}>1
$$

Then $\bar{f} \in F P\left(\mathbf{H}^{\prime}\right)$ is a certificate that the constraint $\bar{y} x \leq 1$ is not valid for $F P\left(\mathbf{H}^{\prime}\right)$. So add $\bar{f}$ to set $F$ and start over - solve a new instance of LP1, find a new constraint $\bar{y} x \leq 1$, and test its validity against the forests of $\mathbf{H}^{\prime}$. If the optimal solution of IP1 has objective $Q(f) \leq 1$, then this serves as a certificate to the validity of constraint $\bar{y} x \leq 1$ with respect to polytope $F P\left(\mathbf{H}^{\prime}\right)$. This constraint lifts directly back into the larger space of $\operatorname{STP}(\mathbf{H})$ as follows. Let $\mathbf{e}^{\prime} \in \mathbf{E}^{\prime}$. Copy the coefficient $\bar{y}_{\mathbf{e}^{\prime}}$ to every edge $\mathbf{e} \in \mathbf{E}$ such that $\mathbf{e} \cap S=\mathbf{e}^{\prime}$. ( $\left|\mathbf{e}^{\prime}\right| \geq 2$ by definition.) Let the coefficient be zero for every edge $\mathbf{e} \in \mathbf{E}$ such that $|\mathbf{e} \cap S| \leq 1$. Note that it is not always necessary to obtain "optimal" solutions for subproblem IP1 - any solution $f$ having $Q(f)>1$ suffices. We use greedy heuristics to try and find a suitable forest $f$. Only when these heuristics fail do we resort to invoking the branch-and-cut recursively to solve IP1 (after reducing 
it to MST in $\mathbf{H}^{\prime \prime}$ ). This recursive invocation of the branch-and-cut terminates early (before proving optimality) whenever a forest $f$ satisfying $Q(f)>1$ is discovered.

This procedure can be quite efficient when $\operatorname{dim}\left(\mathbf{H}^{\prime}\right) \ll \operatorname{dim}(\mathbf{H})$, meaning that we must focus on making $|S|$ sufficiently small. We apply this procedure only to LP solutions having no violated subtour constraints. The separation routines for subtour constraints perform various reductions on the support hypergraph before the formal flow-based separation algorithms are applied. When no subtour violations are present, many of these same reductions can be applied to arrive at "fractional components" upon which generation of local cuts might be attempted. By default, local cuts are attempted on any component for which the induced $\mathbf{H}^{\prime}=\left(S, \mathbf{E}^{\prime}\right)$ satisfies $|S| \leq 80$ and $\left|\mathbf{E}^{\prime}\right| \leq 256$.

These cuts can be very strong in practice. It is not unusual for a single round of local cuts to close $10 \%$ or more of the gap at the root node. Rounds are repeated until either the gap is closed or no further local cuts can be generated (either because the $\bar{x}^{\prime}$ is already a convex combination of forests, or because the components become too big to attempt local cuts upon). The default settings for maximum $|S|$ and $\left|\mathbf{E}^{\prime}\right|$ are a tradeoff between the desire to obtain strong cuts versus the cost of obtaining them, versus the effectiveness of branching. It is useful to remember that expending large amounts of CPU time to compute a single cut does not automatically make that cut "strong."

\section{Computational Experience}

In this section we present our computational results. First we describe the experimental setup. Then we describe the performance of the best configuration, GeoSteiner 4.0 using FST pruning, on three sets of benchmark instances. Then we compare some older code bases to the most recent one; the goal is to illustrate the algorithmic improvements of the GeoSteiner software package.

\subsection{Experimental Setup}

The computational benchmarking was performed on twelve paravirtualized guests sharing a HP ProLiant BL685c generation 7 server. This model has 256GB of memory and four AMD Opteron 6380 CPUs, each having sixteen cores running at a base clock rate of $2.5 \mathrm{GHz}$. The twelve guests were running the 64 -bit version of Debian 7.4.0 while the host was running OpenSuse 12.3. CPLEX version 12.5.1 was used as LP solver. The source code was compiled with GNU C 4.4.5-8 for Debian, with optimization flag -O2.

For the generation of Euclidean FSTs, we use the $-\mathrm{g}$ and $-\mathrm{m} 2$ flags. These indicate, respectively, that the Zachariasen-Winter greedy heuristic (see Section 3.2) should be used instead of the Smith-Lee-Liebman heuristic, and that precision should be increased by using GMP Level 2 (see Section 3.3). For solving the FST concatenation problem, the -L flag was used, which enables the usage of local cuts (see Section 3.11). 
No other parameters were used in the benchmark runs. As a general rule, each of the three phases - FST generation, FST pruning and FST concatenation was allowed to run for at most 24 hours for each problem instance. Exceptions are discussed below.

In the tables below, the names of the columns is identical, and they are defined as follows:

\begin{tabular}{l|l|l}
\hline FST counts & $\begin{array}{l}\text { Gen } \\
\text { Prun } \\
\text { Req }\end{array}$ & $\begin{array}{l}\text { Number of generated FSTs } \\
\text { Number of FSTs after FST pruning } \\
\text { Number of required FSTs identified during FST pruning } \\
\text { (FSTs that belong to any minimum Steiner tree) }\end{array}$ \\
\hline SMT properties & $\begin{array}{l}\text { NumF } \\
\text { SizeF } \\
\text { Red }\end{array}$ & $\begin{array}{l}\text { Number of FSTs in minimum Steiner tree } \\
\text { Average size of FSTs in minimum Steiner tree } \\
\text { Reduction over minimum spanning tree (in percent) }\end{array}$ \\
\hline FST conc & $\begin{array}{l}\text { Gap } \\
\text { Nodes } \\
\text { LPs }\end{array}$ & $\begin{array}{l}\text { Root LP value vs. optimal value (gap in percent) } \\
\text { Number of bPs solved }\end{array}$ \\
\hline CPU time & $\begin{array}{l}\text { Gen } \\
\text { Prun }\end{array}$ & $\begin{array}{l}\text { CPU time for FST generation (seconds) } \\
\text { CPU time for FST pruning (seconds) }\end{array}$ \\
& Conc \\
Total & $\begin{array}{l}\text { CPU time for FST concatenation (seconds) } \\
\text { Total CPU time for FST generation, FST pruning } \\
\text { and FST concatenation (seconds). }\end{array}$ \\
\hline
\end{tabular}

\subsection{Benchmark Instances}

The benchmark instances are divided into three main sets.

1. The rand_points set consists of instances with of size $1000,2000, \ldots, 10,000$, with 15 randomly generated instances of each size. These were generated using the rand_points program of the GeoSteiner package (randomly and uniformly distributed points in a square).

2. The estein set from the OR-Library [3] consists of 31 randomly generated instances: 15 containing 500 terminals, 15 containing 1000 terminals and a single instance containing 10,000 terminals. All instances are randomly and uniformly distributed points in a square.

3. The TSPLIB set consists of 45 problems from TSPLIB, a library of instances for the traveling salesman problem which are mainly drawn from the real world. This benchmark sets contain all instances with 500 or more terminals used in [24] and [20] (plus the smaller TSPLIB instances from [24] for comparison purposes).

All problem instances were solved under four different metrics: the Euclidean metric, the rectilinear metric (two uniform orientations), the hexagonal metric (three uniform orientations) and the octilinear metric (four uniform orientations). 
Euclidean

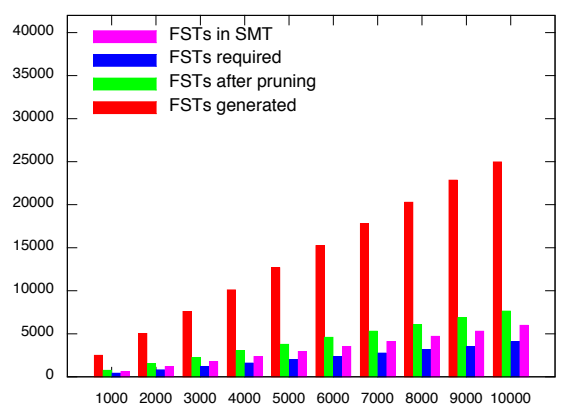

Hexagonal

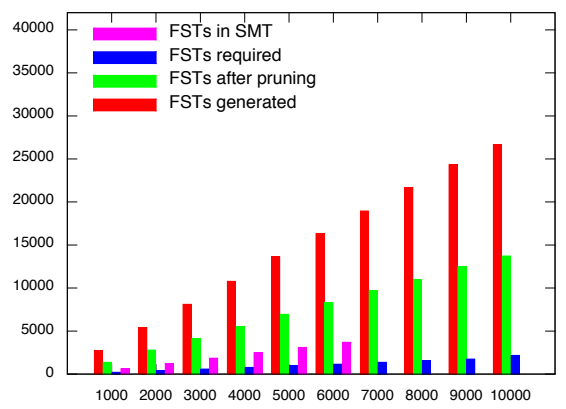

Rectilinear

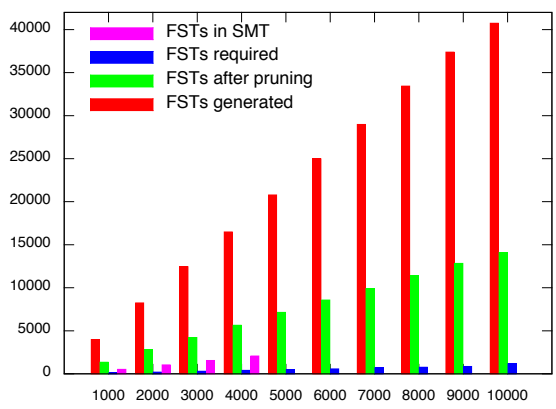

Octilinear

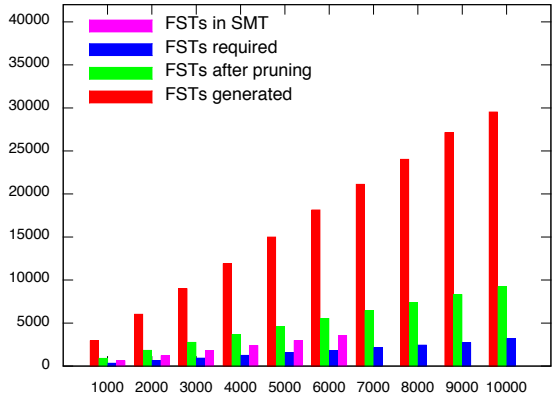

Fig. 2. FST counts for randomly generated problem instances. Averages taken over 15 problem instances for each size.

\subsection{Performance of Best Configuration}

In this section we report the computational performance using the best configuration of the code, namely GeoSteiner 4.0 using FST pruning. The computational results are presented in Figures 2 to 4 and in Tables 2 to 13.

FST counts and sizes For randomly generated problem instances, the number of FSTs follows a very regular pattern (see Figure 2 and Tables 2-9). For the Euclidean problem, around 2.50n FSTs are generated for $n$ terminals; after pruning around $0.76 n$ FSTs remain, and among these $0.41 n$ FSTs have been identified as required FSTs. Thus FST pruning removes more than two-thirds of the FSTs that were originally generated, and only $0.35 n$ FSTs remain undecided in the FST concatenation problem. A minimum Steiner tree has around 0.60n FSTs, so approximately $0.19 n$ FSTs need to be selected among the $0.35 n$ undecided FSTs.

For the rectilinear problem, the FST counts are somewhat higher, but they are still linear in $n$ : around $4.07 n$ FSTs are generated, $1.41 n$ remain after FST pruning, and $0.12 n$ FSTs are identified as required; this leaves $1.29 n$ undecided 
FSTs. For the hexogonal and octilinear metrics, the FST counts are sandwiched between the FST counts for the Euclidean and rectilinear metrics.

The average size of FSTs in a minimum Steiner tree is 2.63 for the hexagonal metric, 2.70 for both the Euclidean and octilinear metrics, and 2.94 for the rectilinear metric. The larger number of generated FSTs for the rectilinear metrics can therefore be explained by the fact that FSTs in a rectilinear minimum Steiner tree span slightly more terminals on average when compared to the other metrics.

For the TSPLIB instances the FST counts vary significantly, depending on the distribution of the terminals. For the Euclidean problem, problem instances where the terminals are aligned on grid lines are particularly difficult, e.g. pcb442 and d1291 for which the number of generated FSTs is significantly larger than the average for randomly generated problem instances. Problem instance p654, which has its points partly aligned on grid lines and partly in clusters, is the smallest unsolved problem for the Euclidean and hexagonal metrics, and it was also somewhat hard for the octilinear metric (but completely trivial for the rectilinear metric).

Branch-and-cut performance The LP-relaxation of the integer programming formulation for the minimum spanning tree in hypergraph problem provides excellent lower bounds for the problem. In most cases the gap between the LPsolution and the IP-solution is zero, and the problem is solved at the root node. Among the TSPLIB instances the largest gap is $0.084 \%$, and the largest number of branch-and-bound nodes is 661 .

The number of LPs solved is basically proportional to the running time of the branch-and-cut algorithm. In Figure 3 we present plots showing the number of LPs solved for the randomly generated problem instances. Several of the larger problem instances did not solve within the time limit of 24 hours, and therefore no data is shown for these problem instances. For the Euclidean metric, all problem instances were solved within 1,000 LP-iterations. For the remaining metrics, some instances required 100,000 or more iterations.

Running times The running time for the randomly generated problem instances, divided into each of the three phases FST generation, FST pruning and FST concatenation, is shown in Figure 4. For the Euclidean metric, all problem instances were solved within the time limit of 24 hours. For the rectilinear metric, all problem instances of size 4,000 or less were solved within the time limit; for the hexagonal and octilinear metrics, all problem instances of size 6,000 or less were solved within the time limit.

The FST generation phase dominates the total running time for the smaller problem instances under all metrics except the rectilinear metric. The running time of FST generation and FST pruning scales in a fairly regular way, and is approximately quadratic. For larger problem instances, FST concatenation dominates the total running time for all metrics. 

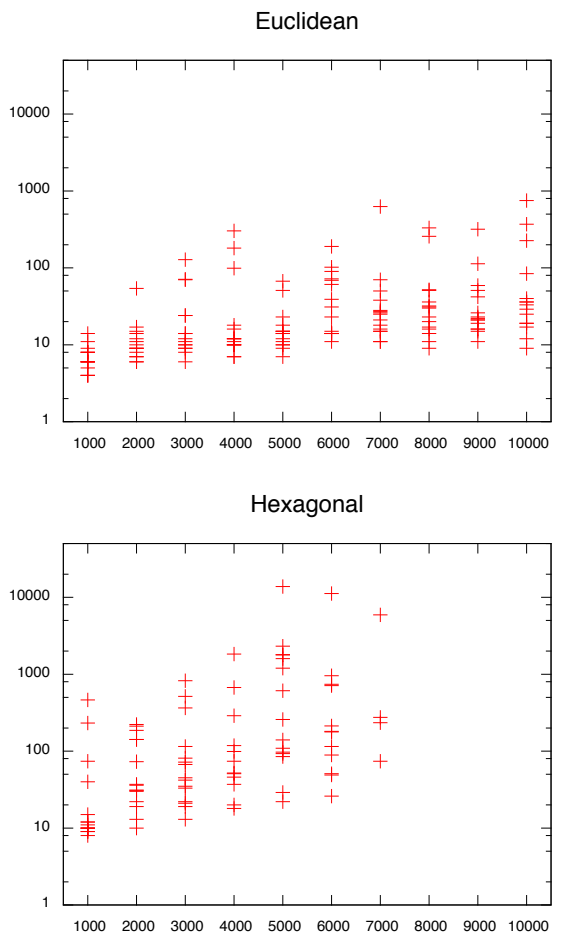

Rectilinear

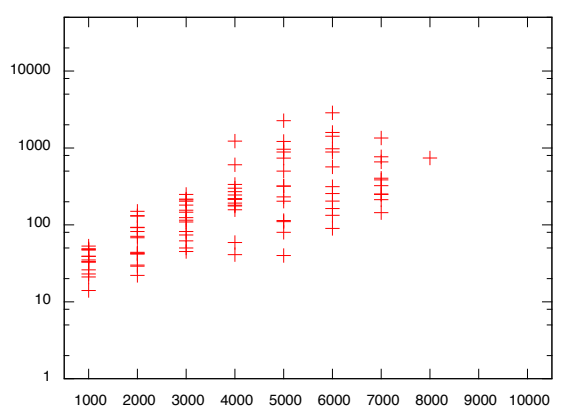

Octilinear

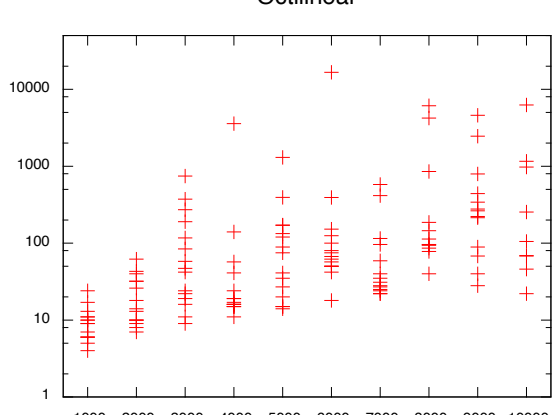

Fig. 3. Number of LPs solved in branch-and-cut algorithm for randomly generated problem instances. 
Euclidean

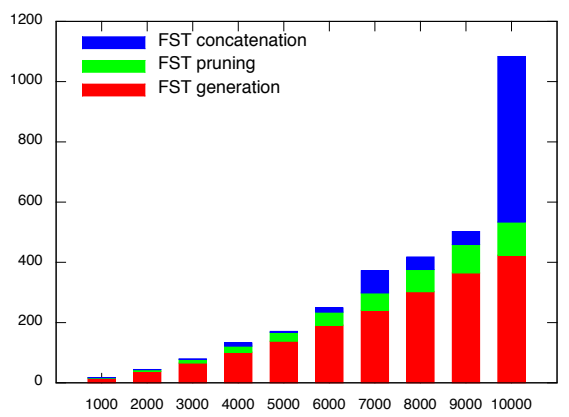

Hexagonal

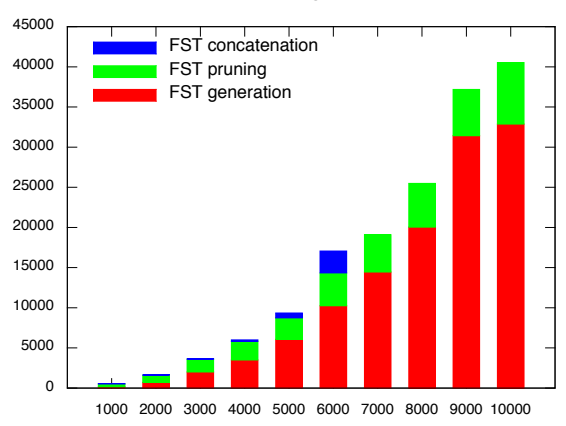

Rectilinear

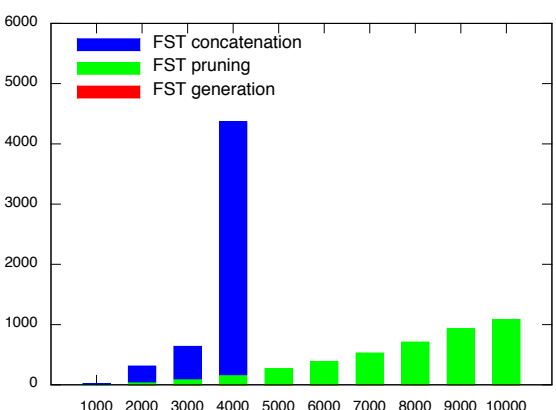

Octilinear

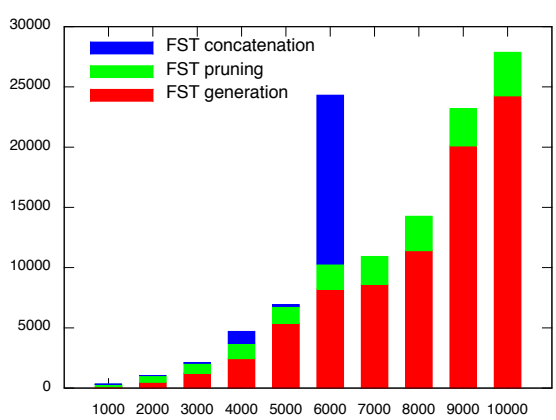

Fig. 4. Running times for randomly generated problem instances. Averages taken over 15 problem instances for each size. The scaling on the y-axis differs across the four figures. The FST concatenation problem was not solved for all of the larger rectilinear, hexagonal and octilinear problem instances - hence no average running times are shown for the larger problem instances.

For the TSPLIB instances (Tables 10-13), we allowed some of the smaller problem instances to run for more than 24 hours. When compared to the 2000study, the following unsolved Euclidean problem instances have now been solved: pcb442, p654 (FST generation and pruning only), d1291, pcb3038, fnl4461 and r15934. The following unsolved rectilinear problem instance have been solved: pcb1173, pcb3038, r15934 and pla7397.

\subsection{Performance Across Code Bases and Configurations}

The effect of the algorithmic enhancements is illustrated by comparing the branch-and-bound code on three different code bases: the code used in the 2000study (GeoSteiner 2000), the publicly available GeoSteiner 3.1 code and the commercial GeoSteiner 4.0 code. Also, we show the effect of using FST pruning in all three cases. 
The tests are made on the 15 estein instances with 1000 terminals. These problem instances were difficult to solve as rectilinear problems using GeoSteiner 2000 , and therefore they nicely illustrate the improvements made. The average running time in the 2000-study on these 15 instances was around 2,000 seconds for the Euclidean metric and more than 100,000 seconds for the rectilinear metric. We estimate that the machine used in the 2000-study was around 15-20 times slower than the one used in current paper.

Table 1 presents the results of running the old and new code on the 15 estein instances (on the same modern machine). It follows from the table that the algorithmic enhancements have resulting in two orders of magnitude speed-up for the rectilinear problem — and somewhat less for the Euclidean problem. FST pruning has a significant effect on the performance of the FST concatenation algorithm.

\begin{tabular}{l|l|l|r|r|r}
\hline Version & Metric & Pruning & Nodes & LPs & Time \\
\hline 2000 & Euclidean & No & 1.3 & 620.1 & 322.74 \\
3.1 & Euclidean & No & 1.0 & 480.1 & 209.61 \\
4.0 & Euclidean & No & 1.0 & 41.7 & 11.73 \\
\hline 2000 & Euclidean & Yes & 1.1 & 23.4 & 17.50 \\
3.1 & Euclidean & Yes & 1.0 & 21.7 & 17.57 \\
4.0 & Euclidean & Yes & 1.0 & 5.1 & 12.43 \\
\hline 2000 & Rectilinear & No & 142.7 & 2857.5 & 7898.26 \\
3.1 & Rectilinear & No & 4.7 & 1015.1 & 1844.88 \\
4.0 & Rectilinear & No & 5.3 & 216.9 & 85.25 \\
\hline 2000 & Rectilinear & Yes & 43.5 & 4405.5 & 2803.18 \\
3.1 & Rectilinear & Yes & 2.9 & 104.1 & 69.26 \\
4.0 & Rectilinear & Yes & 3.0 & 40.7 & 22.10 \\
\hline
\end{tabular}

Table 1. Comparison of different code bases. FST concatenation on the 15 estein instances of size 1000. Average number of nodes, LPs and running times for FST concatenation under the Euclidean and rectilinear metric. FSTs are generated and pruned using GeoSteiner 4.0 for all FST concatenation algorithms. Local cuts were not activated in the GeoSteiner 4.0 code.

\section{Conclusions}

In this paper we presented an updated computational study of the GeoSteiner software package. As a consequence of a number of algorithmic enhancements, the software package can now compute minimum Steiner trees for 5-10 times as many terminals when compared to the 2000-study. Highly structured and/or clustered terminal sets still remain a challenge under some metrics. 


\section{References}

[1] E. Althaus. Berechnung optimaler Steinerbäume in der ebene. Master's thesis, Max-Planck-Institut für Informatik in Saarbrücken, Universität des Saarlandes, 1998.

[2] D. Applegate, R. Bixby, V. Chvátal, and W. Cook. TSP cuts which do not conform to the template paradigm. In Computational Combinatorial Optimization, Optimal or Provably Near-Optimal Solutions, pages 261-304, 2001.

[3] J. E. Beasley. OR-library: Distributing test problems by electronic mail. Journal of the Operational Research Society, 41:1069-1072, 1990.

[4] M. Brazil, D. A. Thomas, J. F. Weng, and M. Zachariasen. Canonical forms and algorithms for Steiner trees in uniform orientation metrics. Algorithmica, 44:281-300, 2006.

[5] M. Brazil and M. Zachariasen. Steiner trees for fixed orientation metrics. Journal of Global Optimization, 43:141-169, 2009.

[6] M. Brazil and M. Zachariasen. Computational complexity for uniform orientation Steiner tree problems. In Proceedings of the Thirty-Sixth Australasian Computer Science Conference (ACSC), 2013.

[7] M. Brazil and M. Zachariasen. Optimal Interconnection Trees in the Plane: Theory, Algorithms and Applications. Springer, 2015 (to appear).

[8] U. Fößmeier and M. Kaufmann. Solving rectilinear Steiner tree problems exactly in theory and practice. In R. Burkard and G. Woeginger, editors, Algorithms ESA 97, volume 1284 of Lecture Notes in Computer Science, pages 171-185. Springer Berlin Heidelberg, 1997.

[9] M. R. Garey, R. L. Graham, and D. S. Johnson. The complexity of computing Steiner minimal trees. SIAM Journal on Applied Mathematics, 32(4):835-859, 1977.

[10] M. R. Garey and D. S. Johnson. The rectilinear Steiner tree problem is NPcomplete. SIAM Journal on Applied Mathematics, 32(4):826-834, 1977.

[11] E. N. Gilbert and H. O. Pollak. Steiner minimal trees. SIAM Journal on Applied Mathematics, 16(1):1-29, 1968.

[12] M. Hanan. On Steiner's problem with rectilinear distance. SIAM Journal on Applied Mathematics, 14(2):255-265, 1966.

[13] T. Huang and E. F. Y. Young. Obsteiner: An exact algorithm for the construction of rectilinear Steiner minimum trees in the presence of complex rectilinear obstacles. Computer-Aided Design of Integrated Circuits and Systems, IEEE Transactions on, 32(6):882-893, 2013.

[14] F. K. Hwang and D. S. Richards. Steiner tree problems. Networks, 22:55-89, 1992.

[15] F. K. Hwang, D. S. Richards, and P. Winter. The Steiner Tree Problem. Annals of Discrete Mathematics 53. Elsevier Science Publishers, Netherlands, 1992.

[16] A. B. Kahng, I. I. Mandoiu, and A. Z. Zelikovsky. Highly scalable algorithms for rectilinear and octilinear Steiner trees. In Proceedings of the Asia-Pacific Design Automation Conferrence, pages 827-833, 2003.

[17] K. Mehlhorn and S. Näher. LEDA: a library of efficient data types and algorithms. In Proceedings of the Seventeenth International Colloquim on Automata, Languages and Programming, pages 1-5, 1990.

[18] K. Mehlhorn and S. Näher. LEDA - A Platform for Combinatorial and Geometric Computing. Max-Planck-Institut für Informatik, Saarbrücken, Germany, http://www.mpi-sb.mpg.de/LEDA/leda.html, 1996. 
[19] B. K. Nielsen, P. Winter, and M. Zachariasen. An exact algorithm for the uniformly-oriented Steiner tree problem. In Proceedings of the 10th European Symposium on Algorithms, Lecture Notes in Computer Science, volume 2461, pages 760-772. Springer, 2002.

[20] T. Polzin and S. Vahdati Daneshmand. On Steiner trees and minimum spanning trees in hypergraphs. Operations Research Letters, 31:12-20, 2003.

[21] J. S. Salowe and D. M. Warme. Thirty-five-point rectilinear Steiner minimal trees in a day. Networks, 25(2):69-87, 1995.

[22] J. M. Smith, D. T. Lee, and J. S. Liebman. An $O(n \log n)$ Heuristic for Steiner Minimal Tree Problems on the Euclidean Metric. Networks, 11:23-29, 1981.

[23] D. M. Warme. Spanning Trees in Hypergraphs with Applications to Steiner Trees. PhD thesis, Computer Science Dept., The University of Virginia, 1998.

[24] D. M. Warme, P. Winter, and M. Zachariasen. Exact algorithms for plane Steiner tree problems: A computational study. In D.-Z. Du, J. M. Smith, and J. H. Rubinstein, editors, Advances in Steiner Trees, pages 81-116. Kluwer Academic Publishers, Boston, 2000.

[25] P. Widmayer, Y. F. Wu, and C. K. Wong. On some distance problems in fixed orientations. SIAM Journal on Computing, 16(4):728-746, 1987.

[26] P. Winter and M. Zachariasen. Euclidean Steiner minimum trees: An improved exact algorithm. Networks, 30:149-166, 1997.

[27] M. Zachariasen. Rectilinear full Steiner tree generation. Networks, 33:125-143, 1999.

[28] M. Zachariasen and A. Rohe. Rectilinear group Steiner trees and applications in VLSI design. Mathematical Programming, 94:407-433, 2003.

[29] M. Zachariasen and P. Winter. Concatenation-based greedy heuristics for the Euclidean Steiner tree problem. Algorithmica, 25:418-437, 1999.

[30] M. Zachariasen and P. Winter. Obstacle-avoiding Euclidean Steiner trees in the plane: An exact algorithm. In Workshop on Algorithm Engineering and Experimentation (ALENEX), Lecture Notes in Computer Science 1619, pages 282-295. Springer, 1999. 
XXII

\begin{tabular}{|c|c|c|c|c|c|c|c|c|c|c|c|c|c|}
\hline \multirow[b]{2}{*}{ Instance } & \multicolumn{3}{|c|}{ FST counts } & \multicolumn{3}{|c|}{ SMT properties } & \multicolumn{3}{|c|}{ FST conc } & \multicolumn{4}{|c|}{ CPU time } \\
\hline & Gen & Prun & Req & NumF & SizeF & Red & Gap & Nodes & LPs & Gen & Prun & Conc & Total \\
\hline \multirow[t]{2}{*}{1000} & 2490 & 751 & 415 & 593 & 2.68 & 3.19 & 0.000 & 1.0 & 7.0 & 15.0 & 2.1 & 0.1 & 17.2 \\
\hline & \pm 51 & \pm 28 & \pm 20 & \pm 8 & \pm 0.03 & \pm 0.11 & \pm 0.000 & \pm 0.0 & \pm 2.8 & \pm 1.1 & \pm 0.2 & \pm 0.0 & \pm 1.2 \\
\hline \multirow[t]{2}{*}{2000} & 5036 & 1504 & 803 & 1178 & 2.70 & 3.29 & 0.000 & 1.0 & 12.9 & 37.6 & 6.1 & 0.5 & 44.3 \\
\hline & \pm 114 & \pm 36 & \pm 44 & \pm 19 & \pm 0.03 & \pm 0.11 & \pm 0.000 & \pm 0.0 & \pm 11.8 & \pm 3.8 & \pm 0.5 & \pm 0.4 & \pm 4.2 \\
\hline \multirow[t]{2}{*}{3000} & 7594 & 2247 & 1202 & 1759 & 2.71 & 3.30 & 0.000 & 1.0 & 28.0 & 65.6 & 11.8 & 2.3 & 79.7 \\
\hline & \pm 102 & \pm 35 & \pm 39 & \pm 16 & \pm 0.02 & \pm 0.06 & \pm 0.000 & \pm 0.0 & \pm 34.7 & \pm 3.4 & \pm 0.8 & \pm 3.0 & \pm 6.1 \\
\hline \multirow[t]{2}{*}{4000} & 10093 & 3018 & 1582 & 2348 & 2.70 & 3.27 & 0.000 & 1.0 & 47.9 & 101.6 & 20.2 & 10.7 & 132.5 \\
\hline & \pm 137 & \pm 43 & \pm 41 & \pm 16 & \pm 0.01 & \pm 0.06 & \pm 0.000 & \pm 0.0 & \pm 85.2 & \pm 8.0 & \pm 1.1 & \pm 23.0 & \pm 27.6 \\
\hline \multirow[t]{2}{*}{5000} & 12661 & 3773 & 1977 & 2929 & 2.71 & 3.29 & 0.000 & 1.0 & 19.4 & 138.0 & 29.6 & 3.0 & 170.6 \\
\hline & \pm 169 & \pm 71 & \pm 59 & \pm 29 & \pm 0.02 & \pm 0.07 & \pm 0.000 & \pm 0.0 & \pm 16.8 & \pm 5.2 & \pm 1.0 & \pm 3.0 & \pm 7.0 \\
\hline \multirow[t]{2}{*}{6000} & 15240 & 4577 & 2333 & 3520 & 2.71 & 3.30 & 0.000 & 1.0 & 50.6 & 191.5 & 43.0 & 14.4 & 248.9 \\
\hline & \pm 178 & \pm 74 & \pm 76 & \pm 25 & \pm 0.01 & \pm 0.05 & \pm 0.000 & \pm 0.0 & \pm 49.3 & \pm 10.8 & \pm 3.1 & \pm 16.0 & \pm 22.7 \\
\hline \multirow[t]{2}{*}{7000} & 17794 & 5294 & 2752 & 4098 & 2.71 & 3.32 & 0.000 & 1.0 & 66.5 & 240.8 & 57.7 & 73.5 & 372.0 \\
\hline & \pm 211 & \pm 62 & \pm 92 & \pm 35 & \pm 0.01 & \pm 0.06 & \pm 0.000 & \pm 0.0 & \pm 155.9 & \pm 12.2 & \pm 3.1 & \pm 259.6 & \pm 257.1 \\
\hline \multirow[t]{2}{*}{8000} & 20278 & 6053 & 3159 & 4702 & 2.70 & 3.30 & 0.000 & 1.0 & 62.0 & 302.5 & 73.6 & 41.6 & 417.7 \\
\hline & \pm 175 & \pm 87 & \pm 75 & \pm 30 & \pm 0.01 & \pm 0.04 & \pm 0.000 & \pm 0.0 & \pm 96.1 & \pm 8.7 & \pm 3.0 & \pm 100.9 & \pm 102.7 \\
\hline \multirow[t]{2}{*}{9000} & 22840 & 6850 & 3520 & 5287 & 2.70 & 3.27 & 0.000 & 1.0 & 51.5 & 364.9 & 94.1 & 43.1 & 502.0 \\
\hline & \pm 149 & \pm 111 & \pm 97 & \pm 25 & \pm 0.01 & \pm 0.04 & \pm 0.000 & \pm 0.0 & \pm 78.2 & \pm 15.5 & \pm 6.6 & \pm 114.0 & \pm 109.3 \\
\hline \multirow[t]{2}{*}{10000} & 24966 & 7632 & 4071 & 5978 & 2.68 & 3.31 & 0.000 & 1.0 & 113.7 & 423.9 & 109.7 & 550.0 & 1083.5 \\
\hline & \pm 1563 & \pm 235 & \pm 616 & \pm 402 & \pm 0.09 & \pm 0.04 & \pm 0.000 & \pm 0.0 & \pm 202.2 & \pm 66.3 & \pm 16.1 & \pm 1833.5 & \pm 1843.3 \\
\hline
\end{tabular}

Table 2. Euclidean metric. Randomly generated instances. Averages over 15 instances for each size; standard deviations on the second line of each row. 
XXIII

\begin{tabular}{|c|c|c|c|c|c|c|c|c|c|c|c|c|c|}
\hline \multirow[b]{2}{*}{ Instance } & \multicolumn{3}{|c|}{ FST counts } & \multicolumn{3}{|c|}{ SMT properties } & \multicolumn{3}{|c|}{ FST conc } & \multicolumn{4}{|c|}{ CPU time } \\
\hline & Gen & Prun & Req & NumF & SizeF & Red & Gap & Nodes & LPs & Gen & Prun & Conc & Total \\
\hline \multirow[t]{2}{*}{1000} & 3978 & 1344 & 115 & 517 & 2.93 & 11.33 & 0.003 & 1.8 & 35.2 & 0.0 & 13.6 & 2.3 & 16.1 \\
\hline & \pm 98 & \pm 51 & \pm 13 & \pm 10 & \pm 0.04 & \pm 0.26 & \pm 0.009 & \pm 2.2 & \pm 11.6 & \pm 0.0 & \pm 1.5 & \pm 1.4 & \pm 2.3 \\
\hline \multirow[t]{2}{*}{2000} & 8239 & 2820 & 198 & 1031 & 2.94 & $=11.67$ & 0.001 & 1.7 & 71.3 & 0.1 & 47.5 & 264.7 & 312.3 \\
\hline & \pm 291 & \pm 145 & \pm 23 & \pm 16 & \pm 0.03 & \pm 0.24 & \pm 0.003 & \pm 2.6 & \pm 41.0 & \pm 0.0 & \pm 7.4 & \pm 790.7 & \pm 792.6 \\
\hline \multirow[t]{2}{*}{3000} & 12446 & 4225 & 300 & 1544 & 2.94 & $=11.76$ & 0.001 & 1.7 & 134.8 & 0.3 & 99.8 & 537.1 & 637.2 \\
\hline & \pm 335 & \pm 133 & \pm 32 & \pm 20 & \pm 0.03 & \pm 0.16 & \pm 0.002 & \pm 1.2 & \pm 66.0 & \pm 0.0 & \pm 13.0 & \pm 963.5 & \pm 961.8 \\
\hline \multirow[t]{2}{*}{4000} & 16483 & 5645 & 396 & 2060 & 2.94 & $=11.63$ & 0.003 & 7.5 & 295.7 & 0.4 & 168.1 & 4200.9 & 4369.4 \\
\hline & \pm 454 & \pm 212 & \pm 38 & \pm 14 & \pm 0.01 & \pm 0.17 & \pm 0.004 & \pm 7.9 & \pm 288.3 & \pm 0.0 & \pm 21.0 & \pm 11666.0 & \pm 11663.1 \\
\hline \multirow[t]{2}{*}{5000} & 20775 & 7134 & 483 & - & - & - & - & - & - & 0.6 & 267.8 & - & - \\
\hline & \pm 440 & \pm 213 & \pm 41 & & & & & & & \pm 0.0 & \pm 29.3 & & \\
\hline \multirow[t]{2}{*}{6000} & 24981 & 8576 & 565 & - & - & - & - & - & - & 0.9 & 386.4 & - & - \\
\hline & \pm 496 & \pm 237 & \pm 40 & & & & & & & \pm 0.0 & \pm 40.4 & & \\
\hline \multirow[t]{2}{*}{7000} & 28973 & 9914 & 694 & - & - & - & - & - & - & 1.1 & 526.2 & - & - \\
\hline & \pm 614 & \pm 220 & \pm 43 & & & & & & & \pm 0.0 & \pm 60.8 & & \\
\hline \multirow[t]{2}{*}{8000} & 33429 & 11396 & 761 & - & - & - & - & - & - & 1.4 & 708.4 & - & - \\
\hline & \pm 447 & \pm 219 & \pm 41 & & & & & & & \pm 0.0 & \pm 94.1 & & \\
\hline \multirow[t]{2}{*}{9000} & 37375 & 12836 & 857 & $\begin{array}{ll}- & \text { - }\end{array}$ & - & - & - & - & - & 1.8 & 931.3 & - & - \\
\hline & \pm 437 & \pm 230 & \pm 49 & & & & & & & \pm 0.0 & \pm 70.4 & & \\
\hline \multirow[t]{2}{*}{10000} & 40740 & 14097 & 1192 & $\begin{array}{ll}- & - \\
\end{array}$ & - & - & - & - & - & \multirow{2}{*}{$\begin{array}{r}2.1 \\
\pm 0.3\end{array}$} & 1082.7 & - & - \\
\hline & \pm 3394 & \pm 590 & \pm 915 & & & & & & & & \pm 206.8 & & \\
\hline
\end{tabular}

Table 3. Rectilinear metric. Randomly generated instances. Averages over 15 instances for each size; standard deviations on the second line of each row.

\begin{tabular}{|c|c|c|c|c|c|c|c|c|c|c|c|c|c|}
\hline \multirow[b]{2}{*}{ Instance } & \multicolumn{3}{|c|}{ FST counts } & \multicolumn{3}{|c|}{ SMT properties } & \multicolumn{3}{|c|}{ FST conc } & \multicolumn{4}{|c|}{ CPU time } \\
\hline & Gen & Prun & Req & NumF & SizeF & Red & Gap & Nodes & LPs & Gen & Prun & Conc & Total \\
\hline \multirow[t]{2}{*}{1000} & 2692 & 1369 & 203 & 614 & 2.63 & 4.40 & 0.000 & 1.1 & 61.7 & 146.3 & 370.5 & 45.5 & 562.2 \\
\hline & \pm 146 & \pm 49 & \pm 18 & \pm 12 & \pm 0.03 & \pm 0.20 & \pm 0.001 & \pm 0.3 & \pm 125.2 & \pm 27.8 & \pm 118.6 & \pm 164.2 & \pm 258.9 \\
\hline \multirow[t]{2}{*}{2000} & 5397 & 2792 & 392 & 1226 & 2.63 & 4.48 & 0.000 & 1.0 & 80.3 & 702.9 & 918.5 & 4.4 & 1625.8 \\
\hline & \pm 259 & \pm 109 & \pm 40 & \pm 25 & \pm 0.03 & \pm 0.21 & \pm 0.000 & \pm 0.0 & \pm 77.4 & \pm 130.2 & \pm 233.4 & \pm 4.4 & \pm 325.6 \\
\hline \multirow[t]{2}{*}{3000} & 8111 & 4132 & 587 & 1850 & 2.62 & 4.50 & 0.000 & 1.0 & 151.1 & 2008.4 & 1600.5 & 17.7 & 3626.5 \\
\hline & \pm 240 & \pm 81 & \pm 29 & \pm 32 & \pm 0.03 & \pm 0.11 & \pm 0.000 & \pm 0.0 & \pm 234.3 & \pm 274.6 & \pm 306.4 & \pm 32.3 & \pm 517.5 \\
\hline \multirow[t]{2}{*}{4000} & 10776 & 5497 & 776 & 2460 & 2.62 & 4.45 & 0.000 & 1.0 & 275.5 & 3492.0 & 2323.7 & 192.8 & 6026.6 \\
\hline & \pm 321 & \pm 111 & \pm 44 & \pm 26 & \pm 0.02 & \pm 0.13 & \pm 0.000 & \pm 0.0 & \pm 523.1 & \pm 687.0 & \pm 666.8 & \pm 522.0 & \pm 1584.2 \\
\hline \multirow[t]{2}{*}{5000} & 13609 & 6942 & 968 & 3100 & 2.61 & 4.48 & 0.000 & 1.0 & 1596.3 & 6050.3 & 2692.5 & 609.1 & 9351.8 \\
\hline & \pm 351 & \pm 142 & \pm 38 & \pm 39 & \pm 0.02 & \pm 0.11 & \pm 0.000 & \pm 0.0 & \pm 3471.6 & \pm 825.9 & \pm 622.2 & \pm 1001.2 & \pm 1402.4 \\
\hline \multirow[t]{2}{*}{6000} & 16318 & 8298 & 1152 & 3691 & 2.63 & 4.52 & 0.000 & 1.1 & 1208.8 & 10252.2 & 4093.2 & 2716.6 & 17244.7 \\
\hline & \pm 441 & \pm 159 & \pm 61 & \pm 45 & \pm 0.02 & \pm 0.10 & \pm 0.000 & \pm 0.3 & \pm 3161.4 & \pm 1618.6 & \pm 1171.3 & \pm 8207.8 & \pm 8230.8 \\
\hline \multirow[t]{2}{*}{7000} & 18953 & 9693 & 1362 & - & - & - & - & - & - & 14448.6 & 4652.2 & - & - \\
\hline & \pm 310 & \pm 155 & \pm 51 & & & & & & & \pm 1650.1 & \pm 1060.9 & & \\
\hline \multirow[t]{2}{*}{8000} & 21649 & 10999 & 1581 & - & - & - & - & - & - & 20039.4 & 5443.6 & - & - \\
\hline & \pm 408 & \pm 181 & \pm 46 & & & & & & & \pm 2472.6 & \pm 874.7 & & \\
\hline \multirow[t]{2}{*}{9000} & 24311 & 12458 & 1743 & - & - & - & - & - & - & 31418.7 & 5765.9 & - & - \\
\hline & \pm 450 & \pm 162 & \pm 58 & & & & & & & \pm 2883.3 & \pm 1227.9 & & \\
\hline \multirow[t]{2}{*}{10000} & 26680 & 13726 & 2172 & - & - & - & - & - & - & 32871.7 & 7660.6 & - & - \\
\hline & \pm 2153 & \pm 525 & \pm 947 & & & & & & & \pm 13695.0 & \pm 2388.0 & & \\
\hline
\end{tabular}

Table 4. Hexagonal metric. Randomly generated instances. Averages over 15 instances for each size; standard deviations on the second line of each row. 
XXIV

\begin{tabular}{|c|c|c|c|c|c|c|c|c|c|c|c|c|c|}
\hline \multirow[b]{2}{*}{ Instance } & \multicolumn{3}{|c|}{ FST counts } & \multicolumn{3}{|c|}{ SMT properties } & \multicolumn{3}{|c|}{ FST conc } & \multicolumn{4}{|c|}{ CPU time } \\
\hline & Gen & Prun & Req & NumF & SizeF & Red & Gap & Nodes & LPs & Gen & Prun & Conc & Total \\
\hline \multirow[t]{2}{*}{1000} & 2920 & 891 & 321 & 593 & 2.69 & 4.49 & 0.000 & 1.0 & 9.9 & 102.3 & 218.7 & 0.5 & 321.5 \\
\hline & \pm 115 & \pm 29 & \pm 22 & \pm 10 & \pm 0.03 & \pm 0.15 & \pm 0.000 & \pm 0.0 & \pm 5.2 & \pm 5.0 & \pm 40.7 & \pm 1.4 & \pm 44.1 \\
\hline \multirow[t]{2}{*}{2000} & 6017 & 1824 & 625 & 1178 & 2.70 & 4.64 & 0.000 & 1.0 & 22.3 & 479.1 & 564.6 & 0.7 & 1044.5 \\
\hline & \pm 174 & \pm 52 & \pm 40 & \pm 14 & \pm 0.02 & \pm 0.13 & \pm 0.000 & \pm 0.0 & \pm 16.4 & \pm 31.3 & \pm 130.3 & \pm 0.4 & \pm 144.0 \\
\hline \multirow[t]{2}{*}{3000} & 8979 & 2749 & 918 & 1768 & 2.69 & 4.63 & 0.000 & 1.0 & 135.3 & 1229.6 & 830.8 & 63.4 & 2123.9 \\
\hline & \pm 167 & \pm 52 & \pm 38 & \pm 22 & \pm 0.02 & \pm 0.07 & \pm 0.000 & \pm 0.0 & \pm 200.0 & \pm 41.7 & \pm 109.1 & \pm 165.1 & \pm 132.3 \\
\hline \multirow[t]{2}{*}{4000} & 11869 & 3648 & 1237 & 2362 & 2.69 & 4.58 & 0.000 & 1.0 & 266.7 & 2444.3 & 1263.3 & 997.5 & 4705.1 \\
\hline & \pm 241 & \pm 70 & \pm 52 & \pm 18 & \pm 0.01 & \pm 0.07 & \pm 0.000 & \pm 0.0 & \pm 917.2 & \pm 113.5 & \pm 274.9 & \pm 3772.9 & \pm 3753.7 \\
\hline \multirow[t]{2}{*}{5000} & 14990 & 4569 & 1532 & 2945 & 2.70 & 4.62 & 0.000 & 1.0 & 174.7 & 5366.7 & 1412.8 & 155.6 & 6935.0 \\
\hline & \pm 259 & \pm 84 & \pm 67 & \pm 22 & \pm 0.01 & \pm 0.09 & \pm 0.000 & \pm 0.0 & \pm 327.4 & \pm 222.3 & \pm 157.6 & \pm 558.8 & \pm 651.8 \\
\hline \multirow[t]{2}{*}{6000} & 18135 & 5530 & 1812 & 3533 & 2.70 & 4.62 & 0.000 & 1.0 & 1196.5 & 8203.7 & 2092.3 & 14015.6 & 24311.6 \\
\hline & \pm 350 & \pm 115 & \pm 71 & \pm 23 & \pm 0.01 & \pm 0.06 & \pm 0.000 & \pm 0.0 & \pm 4269.7 & \pm 320.1 & \pm 327.3 & \pm 54200.5 & \pm 53944.6 \\
\hline \multirow[t]{2}{*}{7000} & 21111 & 6414 & 2129 & - & - & . & - & - & - & 8598.7 & 2330.2 & - & - \\
\hline & \pm 451 & \pm 83 & \pm 91 & & & & & & & \pm 344.9 & \pm 389.5 & & \\
\hline \multirow[t]{2}{*}{8000} & 24023 & 7331 & 2429 & - & - & - & - & - & - & 11408.2 & 2855.4 & - & - \\
\hline & \pm 355 & \pm 126 & \pm 91 & & & & & & & \pm 372.7 & \pm 409.8 & & \\
\hline \multirow[t]{2}{*}{9000} & 27113 & 8303 & 2698 & - & - & . & - & - & - & 20094.5 & 3084.9 & - & - \\
\hline & \pm 381 & \pm 146 & \pm 86 & & & & & & & \pm 626.0 & \pm 561.1 & & \\
\hline \multirow[t]{2}{*}{10000} & 29517 & 9209 & 3193 & - & - & 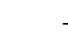 & - & - & - & 24259.5 & 3611.4 & - & - \\
\hline & \pm 2192 & \pm 92 & \pm 726 & & & & & & & \pm 4412.9 & \pm 549.6 & & \\
\hline
\end{tabular}

Table 5. Octilinear metric. Randomly generated instances. Averages over 15 instances for each size; standard deviations on the second line of each row.

\begin{tabular}{|c|c|c|c|c|c|c|c|c|c|c|c|c|c|}
\hline \multirow[b]{2}{*}{ Instance } & \multicolumn{3}{|c|}{ FST counts } & \multicolumn{3}{|c|}{ SMT properties } & \multicolumn{3}{|c|}{ FST conc } & \multicolumn{4}{|c|}{ CPU time } \\
\hline & Gen & Prun & Req & NumF & SizeF & Red & Gap & Nodes & LPs & Gen & Prun & Conc & Total \\
\hline $500(1)$ & 1321 & 370 & 213 & 286 & 2.74 & 3.42 & 0.000 & 1 & 7 & 6.43 & 1.11 & 0.04 & 7.58 \\
\hline $500(2)$ & 1312 & 394 & 181 & 285 & 2.75 & 3.51 & 0.000 & 1 & 13 & 7.07 & 0.90 & 0.10 & 8.07 \\
\hline $500(3)$ & 1321 & 400 & 187 & 288 & 2.73 & 3.37 & 0.000 & 1 & 4 & 6.44 & 1.00 & 0.04 & 7.48 \\
\hline $500(4)$ & 1241 & 373 & 201 & 291 & 2.71 & 3.50 & 0.000 & 1 & 4 & 6.82 & 0.89 & 0.05 & 7.76 \\
\hline $500(5)$ & 1182 & 356 & 238 & 300 & 2.66 & 2.87 & 0.000 & 1 & 7 & 4.91 & 0.70 & 0.06 & 5.67 \\
\hline $500(6)$ & 1308 & 385 & 201 & 287 & 2.74 & 3.37 & 0.000 & 1 & 4 & 7.69 & 1.01 & 0.04 & 8.74 \\
\hline $500(7)$ & 1231 & 382 & 199 & 290 & 2.72 & 3.38 & 0.000 & 1 & 6 & 5.77 & 0.81 & 0.06 & 6.64 \\
\hline $500(8)$ & 1212 & 369 & 211 & 304 & 2.64 & 3.17 & 0.000 & 1 & 5 & 5.78 & 0.76 & 0.04 & 6.58 \\
\hline $500(9)$ & 1279 & 383 & 197 & 294 & 2.70 & 3.38 & 0.000 & 1 & 5 & 6.11 & 0.89 & 0.04 & 7.04 \\
\hline $500(10)$ & 1325 & 379 & 182 & 284 & 2.76 & 3.60 & 0.000 & 1 & 4 & 6.08 & 1.06 & 0.05 & 7.19 \\
\hline $500(11)$ & 1277 & 383 & 208 & 297 & 2.68 & 3.25 & 0.000 & 1 & 2 & 8.25 & 0.86 & 0.03 & 9.14 \\
\hline $500(12)$ & 1218 & 374 & 203 & 302 & 2.65 & 3.21 & 0.000 & 1 & 4 & 6.46 & 0.76 & 0.03 & 7.25 \\
\hline $500(13)$ & 1172 & 348 & 232 & 288 & 2.73 & 3.37 & 0.000 & 1 & 4 & 4.76 & 0.70 & 0.04 & 5.50 \\
\hline $500(14)$ & 1395 & 414 & 160 & 285 & 2.75 & 3.27 & 0.000 & 1 & 33 & 7.83 & 1.11 & 0.18 & 9.12 \\
\hline $500(15)$ & 1268 & 421 & 175 & 294 & 2.70 & 3.22 & 0.000 & 1 & 5 & 6.70 & 0.95 & 0.06 & 7.71 \\
\hline $1000(1)$ & 2435 & 721 & 402 & 580 & 2.72 & 3.45 & 0.000 & 1 & 3 & 14.06 & 1.98 & 0.08 & 16.12 \\
\hline $1000(2)$ & 2533 & 767 & 397 & 587 & 2.70 & 3.40 & 0.000 & 1 & 7 & 13.54 & 2.07 & 0.11 & 15.72 \\
\hline $1000(3)$ & 2424 & 759 & 450 & 609 & 2.64 & 3.17 & 0.000 & 1 & 5 & 17.26 & 1.96 & 0.11 & 19.33 \\
\hline $1000(4)$ & 2676 & 762 & 374 & 572 & 2.75 & 3.30 & 0.000 & 1 & 4 & 14.75 & 2.75 & 0.11 & 17.61 \\
\hline $1000(5)$ & 2413 & 729 & 458 & 604 & 2.65 & 3.10 & 0.000 & 1 & 3 & 14.17 & 1.81 & 0.07 & 16.05 \\
\hline $1000(6)$ & 2525 & 774 & 394 & 582 & 2.72 & 3.23 & 0.000 & 1 & 5 & 14.86 & 2.19 & 0.11 & 17.16 \\
\hline $1000(7)$ & 2454 & 738 & 416 & 587 & 2.70 & 3.26 & 0.000 & 1 & 6 & 14.51 & 2.11 & 0.13 & 16.75 \\
\hline $1000(8)$ & 2533 & 773 & 376 & 586 & 2.70 & 3.42 & 0.000 & 1 & 12 & 15.20 & 2.07 & 0.18 & 17.45 \\
\hline $1000(9)$ & 2603 & 777 & 368 & 576 & 2.73 & 3.37 & 0.000 & 1 & 5 & 16.68 & 2.36 & 0.12 & 19.16 \\
\hline $1000(10)$ & 2472 & 723 & 424 & 598 & 2.67 & 3.36 & 0.000 & 1 & 5 & 13.27 & 1.78 & 0.09 & 15.14 \\
\hline $1000(11)$ & 2532 & 763 & 395 & 583 & 2.71 & 3.14 & 0.000 & 1 & 4 & 14.44 & 2.22 & 0.07 & 16.73 \\
\hline $1000(12)$ & 2616 & 774 & 382 & 570 & 2.75 & 3.58 & 0.000 & 1 & 6 & 16.18 & 2.41 & 0.13 & 18.72 \\
\hline $1000(13)$ & 2488 & 717 & 456 & 591 & 2.69 & 3.19 & 0.000 & 1 & 4 & 14.20 & 2.02 & 0.07 & 16.29 \\
\hline $1000(14)$ & 2522 & 804 & 366 & 587 & 2.70 & 3.48 & 0.000 & 1 & 5 & 15.07 & 2.16 & 0.12 & 17.35 \\
\hline $1000(15)$ & 2531 & 736 & 400 & 576 & 2.73 & 3.24 & 0.000 & 1 & 4 & 15.63 & 2.15 & 0.07 & 17.85 \\
\hline $10000(1)$ & 25125 & 7436 & 4125 & 5905 & 2.69 & 3.29 & 0.000 & 1 & 134 & 416.73 & 06.94 & 31.99 & 555.66 \\
\hline
\end{tabular}

Table 6. Euclidean metric. OR-Library instances. 


\begin{tabular}{|c|c|c|c|c|c|c|c|c|c|c|c|c|c|}
\hline \multirow[b]{2}{*}{ Instance } & \multicolumn{3}{|c|}{ FST counts } & \multicolumn{3}{|c|}{ SMT properties } & \multicolumn{3}{|c|}{ FST conc } & \multicolumn{4}{|c|}{ CPU time } \\
\hline & Gen & Prun & Req & NumF & SizeF & Red & Gap & Nodes & LPs & Gen & Prun & Conc & Total \\
\hline $500(1)$ & 1926 & 626 & 67 & 256 & 2.95 & 11.52 & 0.000 & 1 & 30 & 0.01 & 5.49 & 0.43 & 5.93 \\
\hline $500(2)$ & 2248 & 722 & 54 & 245 & 3.04 & 12.80 & 0.000 & 1 & 16 & 0.02 & 7.01 & 0.28 & 7.31 \\
\hline $500(3)$ & 2155 & 681 & 50 & 248 & 3.01 & 12.40 & 0.000 & 1 & 50 & 0.02 & 5.83 & 0.50 & 6.35 \\
\hline $500(4)$ & 1891 & 693 & 62 & 267 & 2.87 & 11.26 & 0.000 & 1 & 15 & 0.01 & 4.32 & 0.23 & 4.56 \\
\hline $500(5)$ & 1868 & 583 & 67 & 257 & 2.94 & 11.08 & 0.000 & 1 & 41 & 0.01 & 4.04 & 7.54 & 11.59 \\
\hline $500(6)$ & 2069 & 682 & 58 & 262 & 2.90 & 11.69 & 0.000 & 1 & 14 & 0.01 & 5.62 & 0.30 & 5.93 \\
\hline $500(7)$ & 1953 & 604 & 81 & 248 & 3.01 & 11.74 & 0.000 & 1 & 15 & 0.01 & 4.46 & 0.23 & 4.70 \\
\hline $500(8)$ & 2025 & 721 & 55 & 257 & 2.94 & 11.50 & 0.000 & 1 & 38 & 0.02 & 5.07 & 1.15 & 6.24 \\
\hline $500(9)$ & 2010 & 668 & 49 & 263 & 2.90 & 11.15 & 0.000 & 1 & 41 & 0.01 & 5.21 & 4.71 & 9.93 \\
\hline $500(10)$ & 1951 & 619 & 68 & 258 & 2.93 & 11.53 & 0.000 & 1 & 12 & 0.01 & 4.42 & 0.16 & 4.59 \\
\hline $500(11)$ & 2065 & 652 & 68 & 257 & 2.94 & 11.67 & 0.000 & 1 & 20 & 0.01 & 5.76 & 0.33 & 6.10 \\
\hline $500(12)$ & 1912 & 698 & 57 & 260 & 2.92 & 11.21 & 0.000 & 1 & 17 & 0.02 & 5.91 & 0.34 & 6.27 \\
\hline $500(13)$ & 1879 & 657 & 62 & 259 & 2.93 & 11.66 & 0.000 & 1 & 14 & 0.02 & 4.11 & 0.20 & 4.33 \\
\hline $500(14)$ & 2122 & 766 & 40 & 248 & 3.01 & 12.02 & 0.000 & 1 & 37 & 0.02 & 5.67 & 0.69 & 6.38 \\
\hline $500(15)$ & 1953 & 685 & 63 & 261 & 2.91 & 11.22 & 0.000 & 1 & 22 & 0.02 & 5.03 & 0.40 & 5.45 \\
\hline $1000(1)$ & 4164 & 1478 & 83 & 520 & 2.92 & 11.84 & 0.000 & 1 & 57 & 0.04 & 15.93 & 2.63 & 18.60 \\
\hline $1000(2)$ & 4009 & 1335 & 110 & 499 & 3.00 & 11.43 & 0.000 & 1 & 29 & 0.04 & 12.53 & 4.74 & 17.31 \\
\hline $1000(3)$ & 4020 & 1414 & 111 & 521 & 2.92 & 11.16 & 0.000 & 1 & 12 & 0.04 & 14.80 & 0.59 & 15.43 \\
\hline $1000(4)$ & 4090 & 1446 & 92 & 503 & 2.99 & 11.61 & 0.000 & 1 & 28 & 0.04 & 14.18 & 1.16 & 15.38 \\
\hline $1000(5)$ & 4013 & 1388 & 128 & 524 & 2.91 & 11.34 & 0.000 & 1 & 75 & 0.04 & 15.68 & 23.36 & 39.08 \\
\hline $1000(6)$ & 4324 & 1475 & 115 & 507 & 2.97 & 11.57 & 0.000 & 1 & 107 & 0.04 & 17.98 & 35.65 & 53.67 \\
\hline $1000(7)$ & 3996 & 1325 & 110 & 524 & 2.91 & 11.33 & 0.000 & 1 & 26 & 0.04 & 12.98 & 0.83 & 13.85 \\
\hline $1000(8)$ & 4290 & 1461 & 93 & 521 & 2.92 & 11.80 & 0.000 & 1 & 74 & 0.04 & 17.32 & 11.49 & 28.85 \\
\hline $1000(9)$ & 4479 & 1499 & 99 & 504 & 2.98 & 12.10 & 0.000 & 1 & 26 & 0.05 & 19.27 & 1.25 & 20.57 \\
\hline $1000(10)$ & 3992 & 1280 & 101 & 507 & 2.97 & 11.81 & 0.000 & 1 & 109 & 0.04 & 12.44 & 435.76 & 448.24 \\
\hline $1000(11)$ & 4020 & 1426 & 100 & 515 & 2.94 & 11.36 & 0.000 & 1 & 54 & 0.04 & 15.06 & 2.73 & 17.83 \\
\hline $1000(12)$ & 4687 & 1579 & 78 & 493 & 3.03 & 12.71 & 0.001 & 1 & 27 & 0.05 & 26.72 & 6.90 & 33.67 \\
\hline $1000(13)$ & 3873 & 1274 & 132 & 521 & 2.92 & 11.43 & 0.000 & 1 & 18 & 0.04 & 13.19 & 0.57 & 13.80 \\
\hline $1000(14)$ & 4324 & 1478 & 95 & 519 & 2.92 & 11.74 & 0.000 & 1 & 159 & 0.05 & 17.73 & 71.14 & 88.92 \\
\hline $1000(15)$ & 4145 & 1437 & 105 & 514 & 2.94 & 11.58 & 0.000 & 1 & 39 & 0.05 & 15.59 & 2.75 & 18.39 \\
\hline $10000(1)$ & 40878 & 13906 & 935 & 5174 & 2.93 & 11.63 & 0.002 & 19 & 293 & 2.10 & 1183.42 & 738.66 & 1924.18 \\
\hline
\end{tabular}

Table 7. Rectilinear metric. OR-Library instances.

\begin{tabular}{|c|c|c|c|c|c|c|c|c|c|c|c|c|c|}
\hline \multirow[b]{2}{*}{ Instance } & \multicolumn{3}{|c|}{ FST counts } & \multicolumn{3}{|c|}{ SMT properties } & \multicolumn{3}{|c|}{ FST conc } & \multicolumn{4}{|c|}{ CPU time } \\
\hline & Gen & Prun & Req & NumF & SizeF & Red & Gap & Nodes & LPs & Gen & Prun & Conc & Total \\
\hline $500(1)$ & 1280 & 639 & 113 & 304 & 2.64 & 4.71 & 0.000 & 1 & 5 & 28.55 & 109.91 & 0.07 & 138.53 \\
\hline $500(2)$ & 1403 & 744 & 101 & 299 & 2.67 & 4.40 & 0.000 & 1 & 6 & 34.01 & 128.62 & 0.11 & 162.74 \\
\hline $500(3)$ & 1364 & 700 & 102 & 295 & 2.69 & 4.56 & 0.000 & 1 & 5 & 25.03 & 101.54 & 0.08 & 126.65 \\
\hline $500(4)$ & 1364 & 658 & 130 & 311 & 2.60 & 5.01 & 0.000 & 1 & 11 & 30.99 & 147.93 & 0.10 & 179.02 \\
\hline $500(5)$ & 1218 & 656 & 117 & 309 & 2.61 & 4.12 & 0.000 & 1 & 8 & 20.44 & 58.74 & 0.08 & 79.26 \\
\hline $500(6)$ & 1303 & 652 & 121 & 307 & 2.63 & 4.33 & 0.000 & 1 & 71 & 34.11 & 154.02 & 0.43 & 188.56 \\
\hline $500(7)$ & 1292 & 703 & 92 & 320 & 2.56 & 4.74 & 0.000 & 1 & 6 & 23.76 & 92.77 & 0.09 & 116.62 \\
\hline $500(8)$ & 1383 & 691 & 100 & 313 & 2.59 & 4.24 & 0.000 & 1 & 7 & 26.36 & 181.59 & 0.08 & .03 \\
\hline $500(9)$ & 1254 & 650 & 129 & 311 & 2.60 & 4.99 & 0.000 & 1 & 6 & 32 & 94.35 & 8 & .08 \\
\hline $500(10)$ & 1316 & 677 & 88 & 305 & 2. & 4.39 & 0. & 1 & 4 & 3 & 151.32 & 8 & 43 \\
\hline 500 (11) & 1353 & 680 & 93 & 309 & 2.61 & 4.69 & 0.000 & 1 & 9 & 26.81 & 191.68 & 0.13 & 218.62 \\
\hline $500(12)$ & 1201 & 683 & 99 & 324 & 2.54 & 4.12 & 0.000 & 1 & 11 & 18.92 & 98.35 & 0.12 & 117.39 \\
\hline $500(13)$ & 1209 & 672 & 91 & 306 & 2.63 & 4.48 & 0.000 & 1 & 5 & 18.44 & 50.69 & 0.09 & 69.22 \\
\hline $500(14)$ & 1432 & 708 & 81 & 288 & 2.73 & 4.43 & 0.000 & 1 & 24 & & 154.47 & 0.16 & 0.45 \\
\hline $500(15)$ & 1420 & 697 & 103 & 312 & 2.60 & 4.51 & 0.000 & 1 & 6 & 84 & 183.58 & 0.11 & .53 \\
\hline $1000(1)$ & 2799 & 1377 & 200 & 588 & 2 . & 4.58 & 0.000 & 1 & 12 & 22 & 410.31 & 8 & .81 \\
\hline$(2)$ & 2760 & 1349 & 225 & 601 & 2.66 & 4.72 & 0.000 & 1 & 16 & 16 & 276.52 & 0.32 & 5.69 \\
\hline $1000(3)$ & 2456 & 1346 & 240 & 635 & 2.57 & 3.75 & 0.000 & 1 & 33 & 127.66 & 303.06 & 0.48 & 431.20 \\
\hline $1000(4)$ & 2691 & 1365 & 186 & 590 & 2.69 & 4.33 & 0.000 & 1 & 16 & 125.46 & 257.62 & 0.36 & .44 \\
\hline $1000(5)$ & 2597 & 1390 & 195 & 612 & $2 .($ & 4.43 & 0.000 & 1 & 17 & 121 & 266.45 & 0.36 & 20 \\
\hline $1000(6)$ & 2806 & 1464 & 200 & 612 & 2.63 & 4.45 & 0.000 & 1 & 12 & 195 & 385.22 & 0.36 & .75 \\
\hline $1000(7)$ & 2425 & 1265 & 259 & 641 & 2. & 4.26 & 0.000 & 1 & 7 & 50 & 241.33 & 23 & .06 \\
\hline $1000(8)$ & 2844 & 1427 & 169 & 600 & 2. & 4.81 & 0. & 1 & 3106 & 19 & 495.99 & 86.65 & 37 \\
\hline $1000(9)$ & 3041 & 1494 & 172 & 604 & 2.65 & 4.54 & 0.000 & 1 & 22 & 219.68 & 685.77 & 0.46 & 905.91 \\
\hline $1000(10)$ & 2776 & 1400 & 187 & 602 & 2.66 & 4.55 & 0.000 & 1 & 36 & 101.18 & 293.27 & 0.63 & 395.08 \\
\hline $1000(11)$ & 2706 & 1326 & 210 & 632 & 2.58 & 4.49 & 0.000 & 1 & ( & 151.22 & 484.06 & 0.20 & 635.48 \\
\hline $1000(12)$ & 2832 & 1421 & 201 & 608 & 2.64 & 5.02 & 0.000 & 1 & 11 & 172.82 & 328.58 & 0.35 & 501.75 \\
\hline $1000(13)$ & 2497 & 1270 & 215 & 617 & 2.62 & 4.20 & 0.000 & 1 & 10 & 116.71 & 251.36 & 0.23 & 368.30 \\
\hline $1000(14)$ & 2861 & 1402 & 192 & 616 & 2.62 & 4.65 & 0.000 & 1 & 10 & 211.47 & 392.57 & 0.30 & 604.34 \\
\hline & 2725 & 1333 & 190 & 620 & 2.6 & 4.75 & 0.0 & 1 & 12 & 207.58 & 434.25 & 0.31 & 642.14 \\
\hline $10000(1)$ & 26249 & 13785 & 1965 & 174 & 2.62 & 4.52 & 0.000 & 1 & 59 & 27936.87 & 5149.89 & 33.92 & 3120.68 \\
\hline
\end{tabular}

Table 8. Hexagonal metric. OR-Library instances. 
XXVI

\begin{tabular}{|c|c|c|c|c|c|c|c|c|c|c|c|c|}
\hline \multirow[b]{2}{*}{ Instance } & \multicolumn{3}{|c|}{ FST counts } & \multicolumn{2}{|c|}{ SMT properties } & \multicolumn{3}{|c|}{ FST conc } & \multicolumn{4}{|c|}{ CPU time } \\
\hline & Gen & Prun & Req & NumF & SizeF Red & Gap & Nodes & LPs & Gen & Prun & Conc & Total \\
\hline $500(1)$ & 1602 & 425 & 185 & 283 & $2.76 \quad 4.67$ & 0.000 & 1 & 4 & 22.06 & 143.24 & 0.06 & 165.36 \\
\hline $500(2)$ & 1657 & 490 & 139 & 281 & 2.785 .15 & 0.000 & 1 & 12 & 27.41 & 93.65 & 0.10 & 121.16 \\
\hline $500(3)$ & 1666 & 511 & 116 & 284 & $2.76 \quad 4.84$ & 0.000 & 1 & 6 & 25.39 & 93.46 & 0.07 & 118.92 \\
\hline $500(4)$ & 1548 & 465 & 157 & 294 & 2.705 .08 & 0.000 & 1 & 4 & 23.86 & 105.96 & 0.06 & 129.88 \\
\hline $500(5)$ & 1391 & 469 & 166 & 302 & 2.654 .17 & 0.000 & 1 & 7 & 18.96 & 55.68 & 0.06 & 74.70 \\
\hline $500(6)$ & 1547 & 446 & 161 & 294 & $2.70 \quad 4.62$ & 0.000 & 1 & 11 & 23.10 & 128.59 & 0.08 & 151.77 \\
\hline $500(7)$ & 1450 & 462 & 158 & 296 & 2.694 .62 & 0.000 & 1 & 4 & 19.98 & 71.90 & 0.04 & 91.92 \\
\hline $500(8)$ & 1418 & 441 & 160 & 301 & 2.664 .56 & 0.000 & 1 & 6 & 21.05 & 72.98 & 0.06 & 94.09 \\
\hline $500(9)$ & 1426 & 447 & 166 & 300 & 2.664 .48 & 0.000 & 1 & 56 & 21.59 & 72.66 & 0.35 & 94.60 \\
\hline $500(10)$ & 1472 & 451 & 155 & 293 & 2.704 .72 & 0.000 & 1 & 8 & 21.22 & 82.42 & 0.07 & 103.71 \\
\hline $500(11)$ & 1535 & 463 & 141 & 300 & 2.664 .56 & 0.000 & 1 & 7 & 25.51 & 139.05 & 0.06 & 62 \\
\hline $500(12)$ & 1328 & 420 & 181 & 301 & 2.664 .42 & 0.000 & 1 & 4 & 70 & 73.55 & 0.04 & 29 \\
\hline $500(13)$ & 1430 & 422 & 191 & 295 & 2.694 .66 & 0.000 & 1 & 15 & 18.61 & 55.73 & 0.16 & 50 \\
\hline $500(14)$ & 1623 & 501 & 128 & 288 & 2.734 .59 & 0.000 & 1 & 5 & 25.61 & 115.57 & 0.06 & 141.24 \\
\hline $500(15)$ & 1549 & 493 & 143 & 300 & 2.664 .63 & 0.000 & 1 & 44 & 21.60 & 111.09 & 9.69 & .38 \\
\hline $1000(1)$ & 2832 & 882 & 340 & 615 & 2.624 .66 & 0.000 & 1 & 7 & 99.67 & 225.55 & 0.29 & .51 \\
\hline $1000(2)$ & 2973 & 954 & 286 & 586 & 2.704 .81 & 0.000 & 1 & 9 & 00 & 148.82 & 0.19 & .01 \\
\hline $1000(3)$ & 2961 & 952 & 324 & 605 & 2.654 .32 & 0.000 & 1 & 18 & 109 & 357.76 & 0.24 & 03 \\
\hline (4) & 3141 & 934 & 297 & 576 & 2.734 .82 & 0.000 & 1 & 1 & 16 & 200.84 & 0.13 & 13 \\
\hline $1000(5)$ & 2962 & 886 & 368 & 608 & 2.644 .31 & 0.000 & 1 & 8 & 107.23 & 212.93 & 0.14 & 32 \\
\hline $1000(6)$ & 3104 & 914 & 314 & 588 & 2.704 .65 & 0.000 & 1 & 30 & 111.17 & 181.13 & 0.36 & 292.66 \\
\hline $1000(7)$ & 2869 & 902 & 305 & 580 & 2.724 .55 & 0.000 & 1 & 21 & 98.74 & 222.80 & 0.58 & 322.12 \\
\hline $1000(8)$ & 3079 & 923 & 279 & 576 & 2.734 .89 & 0.000 & 1 & 122 & 121.31 & 219.21 & 540.03 & 880.55 \\
\hline $1000(9)$ & 3048 & 965 & 288 & 585 & 2.714 .64 & 0.000 & 1 & 19 & 86 & 235.96 & 0.35 & 355.17 \\
\hline$(10)$ & 2954 & 898 & 315 & 598 & $2.67 \quad 4.74$ & & 1 & 8 & & 183.66 & 0.17 & 28 \\
\hline $1000(11$ & 3029 & 942 & 273 & 587 & 2.704 .54 & 0.000 & 1 & 6 & 05 & 211.09 & 0.15 & 324.29 \\
\hline $1000(12)$ & 3321 & 920 & 312 & 571 & 2.754 .94 & 0.000 & 1 & 8 & 131.34 & 260.39 & 0.17 & 391.90 \\
\hline $1000(13)$ & 2826 & 849 & 356 & 583 & 2.714 .43 & 0.000 & 1 & 5 & 93.60 & 238.36 & 0.10 & 332.06 \\
\hline $1000(14)$ & 3257 & 948 & 292 & 586 & $2.70 \quad 4.88$ & 0.000 & 1 & 9 & 130.79 & 245.74 & 0.16 & 376.69 \\
\hline $1000(15)$ & 2988 & 916 & 290 & 574 & 2.744 .56 & 0.000 & 1 & 5 & 110.72 & 212.12 & 0.15 & 322.99 \\
\hline $10000(1)$ & 29855 & 9091 & 3058 & 5886 & 2.704 .58 & 0.000 & 1 & 134 & 25932.21 & 3441.30 & 30.10 & 29403.61 \\
\hline
\end{tabular}

Table 9. Octilinear metric. OR-Library instances. 


\begin{tabular}{|c|c|c|c|c|c|c|c|c|c|c|c|c|}
\hline \multirow[b]{2}{*}{ Instance } & \multicolumn{3}{|c|}{ FST counts } & \multicolumn{2}{|c|}{ SMT properties } & \multicolumn{3}{|c|}{ FST conc } & \multicolumn{4}{|c|}{ CPU time } \\
\hline & Gen & Prun & Req & NumF & SizeF Red & Gap & Nodes & LPs & Gen & Prun & Conc & Total \\
\hline $\mathrm{d} 198$ & 852 & 264 & 54 & 103 & 2.912 .91 & 0.000 & 1 & 5 & 21.92 & 1.92 & 0.03 & 23.87 \\
\hline $\operatorname{lin} 318$ & 1229 & 442 & 42 & 208 & 2.524 .77 & 0.000 & 1 & 7 & 15.21 & 1.72 & 0.07 & 17.00 \\
\hline fl 417 & 3656 & 2207 & 94 & 180 & $\begin{array}{lll}3.31 & 3.34\end{array}$ & 0.000 & 1 & 6 & 209.87 & 55.44 & 0.25 & 265.56 \\
\hline pcb442 & 8690 & 1771 & 126 & 261 & 2.694 .08 & 0.042 & 13 & 108 & 38917.65 & 1499.18 & 2.54 & 40419.37 \\
\hline att532 & 1382 & 404 & 217 & 310 & 2.713 .36 & 0.000 & 1 & 19 & 13.64 & 1.04 & 0.14 & 14.82 \\
\hline $\operatorname{ali535}$ & 1271 & 416 & 262 & 338 & $2.58 \quad 2.81$ & 0.000 & 1 & 11 & 19.00 & 1.14 & 0.06 & 20.20 \\
\hline u 574 & 1426 & 450 & 230 & 344 & $2.67 \quad 3.15$ & 0.000 & 1 & 10 & 13.03 & 1.10 & 0.09 & 14.22 \\
\hline rat575 & 1651 & 503 & 170 & 323 & $2.78 \quad 3.62$ & 0.000 & 1 & 9 & 15.97 & 1.51 & 0.14 & 17.62 \\
\hline p654 & 29943 & 14458 & 120 & - & - & - & - & - & 882034.39 & 21822.87 & - & - \\
\hline d657 & 1654 & 494 & 277 & 390 & 2.683 .00 & 0.000 & 1 & 6 & 52.12 & 1.83 & 0.07 & 54.02 \\
\hline gr666 & 1763 & 497 & 261 & 378 & $2.76 \quad 3.15$ & 0.000 & 1 & 8 & 56.49 & 1.79 & 0.09 & 58.37 \\
\hline $\mathrm{u} 724$ & 1926 & 515 & 304 & 409 & 2.773 .53 & 0.000 & 1 & 4 & 13.23 & 1.59 & 0.05 & 14.87 \\
\hline rat783 & 2153 & 658 & 232 & 448 & $2.75 \quad 3.52$ & 0.000 & 1 & 7 & 19.32 & 1.95 & 0.12 & 21.39 \\
\hline dsj1000 & 2457 & 719 & 466 & 596 & 2.682 .88 & 0.000 & 1 & 11 & 71.97 & 3.09 & 0.14 & 75.20 \\
\hline pr1002 & 2512 & 721 & 448 & 579 & $2.73 \quad 3.05$ & 0.000 & 1 & 4 & 46.74 & 2.69 & 0.09 & 49.52 \\
\hline u1060 & 3690 & 1470 & 431 & 597 & 2.773 .25 & 0.005 & 37 & 255 & 110.76 & 16.41 & 19.31 & 146.48 \\
\hline vm1084 & 2244 & 915 & 633 & 786 & $2.38 \quad 2.75$ & 0.000 & 1 & 5 & 10.76 & 1.47 & 0.09 & 12.32 \\
\hline pcb1173 & 2941 & 994 & 613 & 802 & 2.463 .18 & 0.000 & 1 & 11 & 43.66 & 5.04 & 0.21 & 48.91 \\
\hline d1291 & 33100 & 3204 & 82 & 996 & 2.302 .78 & 0.027 & 49 & 1040 & 353162.66 & 3092.52 & 177.48 & 6432.66 \\
\hline rl1304 & 2529 & 1204 & 869 & 1058 & 2.231 .68 & 0.000 & 1 & 6 & 15.86 & 2.10 & 0.15 & 18.11 \\
\hline rl1323 & 2500 & 1204 & 883 & 1076 & $2.23 \quad 1.65$ & 0.000 & 1 & 4 & 23.09 & 1.91 & 0.08 & 25.08 \\
\hline nrw1379 & 4105 & 1255 & 345 & 752 & 2.834 .16 & 0.000 & 1 & 9 & 43.83 & 5.88 & 0.39 & 50.10 \\
\hline fl1400 & - & - & & - & - & - & - & - & - & - & - & - \\
\hline 432 & - & - & - & - & - & - & - & - & - & - & - & - \\
\hline fl1577 & - & - & - & - & - & - & - & - & - & - & - & - \\
\hline d1655 & - & - & - & - & - & - & - & - & - & - & - & - \\
\hline vm1748 & 3643 & 1527 & 943 & 1258 & 2.392 .81 & 0.000 & 1 & 6 & 23.10 & 3.43 & 0.23 & 26.76 \\
\hline $\mathrm{u} 1817$ & & - & & - & - & - & - & 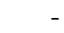 & - & - & - & \\
\hline rl1889 & 3651 & 1683 & 1248 & 1485 & 2.272 .02 & 0.000 & 1 & 4 & 29.53 & 4.06 & 0.19 & 33.78 \\
\hline d2103 & - & - & & - & - & - & - & - & - & - & - & - \\
\hline u2152 & - & - & - & - & - & - & - & - & - & - & - & - \\
\hline u2319 & - & - & - & - & - & - & - & - & - & - & - & - \\
\hline pr2392 & 6373 & 2016 & 987 & 1476 & 2.623 .61 & 0.000 & 1 & 6 & 67.20 & 10.56 & 0.40 & 78.16 \\
\hline pcb3038 & 11636 & 4376 & 914 & 1879 & 2.623 .69 & 0.004 & 13 & 163 & 720.95 & 97.82 & 85.85 & 904.62 \\
\hline fl3795 & & - & & - & - & - & - & & - & - & - & - \\
\hline fnl4461 & 13323 & 4068 & 1091 & 2457 & 2.824 .13 & 0.000 & 1 & 159 & 301.81 & 46.13 & 44.95 & 392.89 \\
\hline rl5915 & 18995 & 7034 & 3479 & 4979 & 2.192 .22 & 0.000 & 1 & 25 & 3025.80 & 136.86 & 4.57 & 3167.23 \\
\hline rl5934 & 18163 & 7239 & 3129 & 4825 & 2.232 .13 & 0.001 & 3 & 232 & 1152.83 & 146.80 & 661.40 & 1961.03 \\
\hline pla7397 & - & - & & - & - & 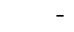 & - & & - & - & - & 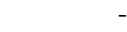 \\
\hline 1849 & - & - & & - & - & - & - & 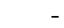 & - & - & - & - \\
\hline usa13509 & 33327 & 10810 & 5586 & - & - & - & - & 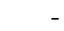 & 3539.85 & 240.38 & - & - \\
\hline brd14051 & 40831 & 12305 & 3986 & 7864 & 2.794 .00 & 0.000 & 1 & 467 & 36234.57 & 665.05 & 3660.68 & 40560.30 \\
\hline $\mathrm{d} 15112$ & 43467 & 12714 & 4465 & - & - & 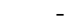 & - & & 6740.17 & 483.48 & - & - \\
\hline d18512 & 54211 & 16447 & 5028 & 10323 & 2.794 .04 & 0.000 & 1 & 1336 & 12045.25 & 834.17 & 122217.99 & 135097.41 \\
\hline pla33810 & - & - & - & - & - & - & - & - & - & - & - & - \\
\hline
\end{tabular}

Table 10. Euclidean metric. TSPLIB instances. 
XXVIII

\begin{tabular}{|c|c|c|c|c|c|c|c|c|c|c|c|c|c|}
\hline \multirow[b]{2}{*}{ Instance } & \multicolumn{3}{|c|}{ FST counts } & \multicolumn{3}{|c|}{ SMT properties } & \multicolumn{3}{|c|}{ FST conc } & \multicolumn{4}{|c|}{ CPU time } \\
\hline & Gen & Prun & Req & NumF & SizeF & Red & Gap & Nodes & LPs & Gen & Prun & Conc & Total \\
\hline $\begin{array}{l}\mathrm{d} 198 \\
\end{array}$ & 265 & 200 & 143 & 173 & 2.14 & 3.66 & 0.000 & 1 & 3 & 0.00 & 0.06 & 0.00 & 0.06 \\
\hline $\operatorname{lin} 318$ & 963 & 492 & 53 & 227 & 2.40 & 8.90 & 0.000 & 1 & 60 & 0.01 & 1.49 & 2694.71 & 2696.21 \\
\hline fl 417 & 1178 & 698 & 127 & 238 & 2.75 & 11.94 & 0.000 & 1 & 75 & 0.01 & 1.75 & 62.01 & 63.77 \\
\hline pcb442 & 558 & 422 & 353 & 394 & 2.12 & 3.99 & 0.000 & 1 & 3 & 0.01 & 0.11 & 0.03 & 0.15 \\
\hline att532 & 2230 & 751 & 78 & 273 & 2.95 & 11.44 & 0.000 & 1 & 222 & 0.01 & 6.97 & 83.95 & 90.93 \\
\hline ali535 & 1788 & 575 & 157 & 308 & 2.73 & 9.77 & 0.000 & 1 & 47 & 0.01 & 3.67 & 0.81 & 4.49 \\
\hline $\mathrm{u} 574$ & 1470 & 586 & 176 & 375 & 2.53 & 8.94 & 0.000 & 1 & 10 & 0.01 & 1.61 & 0.13 & 1.75 \\
\hline rat575 & 3326 & 1217 & 17 & 282 & 3.04 & 13.11 & 0.000 & 1 & 16 & 0.03 & 13.43 & 0.72 & 14.18 \\
\hline p654 & 930 & 668 & 504 & 586 & 2.11 & 5.89 & 0.000 & 1 & 4 & 0.03 & 0.40 & 0.05 & 0.48 \\
\hline d657 & 2259 & 841 & 121 & 379 & 2.73 & 10.57 & 0.000 & 1 & 118 & 0.02 & 4.78 & 4.61 & 9.41 \\
\hline $\operatorname{gr} 666$ & 2804 & 930 & 95 & 349 & 2.91 & 11.03 & 0.000 & 1 & 210 & 0.02 & 10.06 & 47.52 & 57.60 \\
\hline u 724 & 1658 & 813 & 250 & 506 & 2.43 & 9.63 & 0.000 & 1 & 29 & 0.02 & 1.57 & 0.50 & 2.09 \\
\hline rat783 & 3870 & 1432 & 39 & 418 & 2.87 & 12.65 & 0.000 & 1 & 61 & 0.04 & 15.85 & 1.51 & 17.40 \\
\hline dsj1000 & 4086 & 1254 & 136 & 503 & 2.99 & 11.09 & 0.000 & 1 & 25 & 0.04 & 15.13 & 1.10 & 16.27 \\
\hline pr1002 & 2147 & 922 & 459 & 702 & 2.43 & 8.63 & 0.000 & 1 & 11 & 0.04 & 1.81 & 0.86 & 2.71 \\
\hline u1060 & 2765 & 1236 & 327 & 675 & 2.57 & 11.35 & 0.000 & 1 & 145 & 0.04 & 4.48 & 774.91 & 779.43 \\
\hline $\mathrm{vm} 1084$ & 2313 & 1137 & 612 & 838 & 2.29 & 8.35 & 0.000 & 1 & 15 & 0.05 & 2.69 & 0.34 & 3.08 \\
\hline pcb1173 & 2886 & 941 & 408 & 688 & 2.70 & 6.20 & 0.000 & 1 & 12 & 0.04 & 3.58 & 0.40 & 4.02 \\
\hline $\mathrm{d} 1291$ & 1379 & 1334 & 1164 & 1250 & 2.03 & 1.80 & 0.000 & 1 & 4 & 0.06 & 0.41 & 0.06 & 0.53 \\
\hline rl1304 & 1926 & 1254 & 1007 & 1141 & 2.14 & 5.04 & 0.000 & 1 & 7 & 0.05 & 1.07 & 0.14 & 1.26 \\
\hline rl1323 & 1899 & 1287 & 956 & 1157 & 2.14 & 5.45 & 0.000 & 1 & 7 & 0.05 & 1.12 & 0.13 & 1.30 \\
\hline nrw1379 & 8202 & 2864 & 55 & 661 & 3.08 & 13.02 & 0.000 & 1 & 84 & 0.09 & 90.45 & 25.81 & 116.35 \\
\hline fl1400 & 5830 & 3667 & 166 & - & - & & - & - & - & 0.10 & 19.90 & - & - \\
\hline u1432 & 1431 & 1431 & 1431 & 1431 & 2.00 & 0.00 & 0.000 & 1 & 1 & 0.06 & 0.21 & 0.03 & 0.30 \\
\hline fl1577 & 3820 & 2416 & 601 & 1191 & 2.32 & 10.59 & 0.000 & 1 & 11 & 0.08 & 5.82 & 1.29 & 7.19 \\
\hline d1655 & 2129 & 1677 & 1314 & 1508 & 2.10 & 3.57 & 0.000 & 1 & 16 & 0.07 & 1.14 & 0.32 & 1.53 \\
\hline vm1748 & 3912 & 1940 & 945 & 1328 & 2.32 & 8.93 & 0.000 & 1 & 30 & 0.11 & 6.88 & 3.15 & 10.14 \\
\hline $\mathrm{u} 1817$ & 1839 & 1820 & 1787 & 1805 & 2.01 & 0.36 & 0.000 & 1 & 3 & 0.10 & 0.37 & 0.05 & 0.52 \\
\hline rl1889 & 2867 & 1836 & 1355 & 1612 & 2.17 & 5.49 & 0.000 & 1 & 6 & 0.09 & 2.18 & 0.31 & 2.58 \\
\hline $\mathrm{d} 2103$ & 2240 & 2104 & 1964 & 2046 & 2.03 & 0.61 & 0.000 & 1 & 3 & 0.14 & 0.89 & 0.08 & 1.11 \\
\hline $\mathrm{u} 2152$ & 2171 & 2160 & 2123 & 2142 & 2.00 & 0.22 & 0.000 & 1 & 4 & 0.13 & 0.48 & 0.06 & 0.67 \\
\hline $\mathrm{u} 2319$ & 2318 & 2318 & 2318 & 2318 & 2.00 & 0.00 & 0.000 & 1 & 1 & 0.17 & 0.54 & 0.02 & 0.73 \\
\hline pr2392 & 4443 & 2357 & 1204 & 1738 & 2.38 & 7.75 & 0.000 & 1 & 14 & 0.13 & 4.11 & 1.01 & 5.25 \\
\hline pcb3038 & 9048 & 3138 & 706 & 1704 & 2.78 & 6.20 & 0.000 & 1 & 4764 & 0.19 & 30.73 & 16448.87 & 16479.79 \\
\hline fl3795 & 7768 & 5986 & 2727 & - & - & - & - & - & - & 0.41 & 17.96 & - & - \\
\hline fnl4461 & 27818 & 9473 & 163 & - & - & - & - & - & - & 0.54 & 762.73 & - & - \\
\hline rl5915 & 7462 & 5841 & 4989 & 5425 & 2.09 & 3.46 & 0.000 & 1 & 21 & 0.72 & 7.49 & 4.55 & 12.76 \\
\hline rl5934 & 8052 & 5898 & 4762 & 5353 & 2.11 & 3.15 & 0.000 & 1 & 24 & 0.70 & 9.65 & 2.85 & 13.20 \\
\hline pla7397 & 10452 & 7799 & 5608 & 6779 & 2.09 & 3.88 & 0.000 & 1 & 99 & 1.35 & 19.52 & 11.61 & 32.48 \\
\hline rl11849 & 16582 & 11758 & 9069 & 10432 & 2.14 & 3.86 & 0.000 & 1 & 321 & 2.76 & 49.10 & 143.68 & 195.54 \\
\hline usa13509 & 53025 & 18585 & 1804 & - & - & - & - & - & 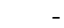 & 2.94 & 1969.08 & - & - \\
\hline brd14051 & 77236 & 27581 & 700 & - & - & - & - & - & - & 3.65 & 7491.68 & - & - \\
\hline $\mathrm{d} 15112$ & 80581 & 28224 & 784 & - & - & - & - & - & - & 4.84 & 7039.53 & - & - \\
\hline $\mathrm{d} 18512$ & 105887 & 37497 & 823 & - & & & - & - & & 6.14 & 15157.44 & 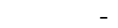 & - \\
\hline pla33810 & 47821 & 35328 & 20269 & 27432 & 2.23 & 2.07 & 0.000 & 3 & 4420 & 22.10 & 404.54 & 5644.07 & 6070.71 \\
\hline
\end{tabular}

Table 11. Rectilinear metric. TSPLIB instances. 


\begin{tabular}{|c|c|c|c|c|c|c|c|c|c|c|c|c|}
\hline \multirow[b]{2}{*}{ Instance } & \multicolumn{3}{|c|}{ FST counts } & \multicolumn{2}{|c|}{ SMT properties } & \multicolumn{3}{|c|}{ FST conc } & \multicolumn{4}{|c|}{ CPU time } \\
\hline & Gen & Prun & Req & NumF & SizeF Red & Gap & Nodes & LPs & Gen & Prun & Conc & Total \\
\hline d198 & 544 & 419 & 26 & 133 & 2.483 .58 & 0.000 & 1 & 55 & 1.38 & 50.51 & 1.08 & 52.97 \\
\hline $\operatorname{lin} 318$ & 1415 & 547 & 58 & 214 & 2.484 .61 & 0.000 & 1 & 7 & 61.00 & 253.12 & 0.11 & 314.23 \\
\hline fl 417 & 1734 & 1067 & 35 & 201 & 3.074 .94 & 0.000 & 1 & 89 & 74.45 & 574.01 & 1.31 & 649.77 \\
\hline pcb442 & 5520 & 1227 & 122 & 296 & 2.495 .35 & 0.084 & 11 & 36 & 850.51 & 15382.63 & 0.88 & 16234.02 \\
\hline att532 & 1521 & 699 & 111 & 327 & 2.624 .83 & 0.000 & 1 & 62 & 34.79 & 257.00 & 0.72 & 292.51 \\
\hline ali535 & 1437 & 773 & 122 & 366 & 2.463 .68 & 0.000 & 1 & 85 & 72.68 & 383.60 & 0.69 & 456.97 \\
\hline u574 & 1338 & 843 & 125 & 368 & 2.563 .40 & 0.000 & 1 & 15 & 13.01 & 165.38 & 0.17 & 178.56 \\
\hline rat575 & 2143 & 1034 & 60 & 323 & $2.78 \quad 5.27$ & 0.000 & 1 & 5 & 173.76 & 323.16 & 0.15 & 497.07 \\
\hline p654 & & & & - & - & - & - & 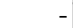 & - & & - & - \\
\hline d657 & 2002 & 1049 & 106 & 391 & $2.68 \quad 4.35$ & 0.000 & 1 & 26 & 52.23 & 790.81 & 0.40 & 843.44 \\
\hline gr666 & 2054 & 959 & 106 & 401 & 2.664 .55 & 0.000 & 1 & 20 & 54.66 & 770.20 & 0.50 & 825.36 \\
\hline u724 & 1717 & 1205 & 120 & 484 & 2.493 .44 & 0.000 & 1 & 11 & 32.39 & 117.40 & 0.23 & 150.02 \\
\hline rat783 & 2593 & 1323 & 91 & 460 & 2.704 .95 & 0.000 & 1 & 22 & 165.36 & 451.75 & 0.45 & 617.56 \\
\hline dsj1000 & 2750 & 1338 & 243 & 610 & 2.643 .97 & 0.000 & 1 & 16 & 130.02 & 1440.26 & 0.42 & 1570.70 \\
\hline pr1002 & 2695 & 1506 & 193 & 645 & 2.554 .28 & 0.000 & 1 & 30 & 58.51 & 1039.10 & 0.57 & 1098.18 \\
\hline u1060 & 3022 & 1790 & 196 & - & - & - & - & - & 107.22 & 1402.49 & - & - \\
\hline vm1084 & 1933 & 1496 & 411 & 845 & $2.28 \quad 2.52$ & 0.000 & 1 & 6 & 26.65 & 98.04 & 0.25 & 124.94 \\
\hline pcb1173 & 3043 & 1555 & 400 & 787 & 2.494 .72 & 0.000 & 1 & 8 & 279.14 & 2751.92 & 0.29 & .35 \\
\hline $\mathrm{d} 1291$ & 5425 & 3986 & 38 & 1034 & 2.253 .65 & 0.000 & 1 & 21 & 837.83 & 4527.74 & 1.69 & 5367.26 \\
\hline rl1304 & 1905 & 1750 & 770 & 1165 & 2.120 .73 & 0.000 & 1 & 18 & 13.77 & 112.27 & 0.55 & 126.59 \\
\hline rl1323 & 2053 & 1763 & 714 & 1154 & $2.15 \quad 1.44$ & 0.000 & 1 & 7 & 19.70 & 225.99 & 0.31 & 246.00 \\
\hline nrw1379 & 6051 & 2740 & 93 & 756 & 2.825 .89 & 0.000 & 1 & 174 & 3368.13 & 2971.76 & 19.13 & 6359.02 \\
\hline fl1400 & 15416 & 7589 & 74 & - & - & - & - & - & 17090.64 & 35574.42 & - & - \\
\hline u1432 & & & & - & - & - & - & - & - & & - & - \\
\hline fl1577 & 7229 & 5509 & 324 & 1228 & 2.283 .42 & 0.000 & 1 & 11 & 17501.08 & 26926.86 & 3.91 & 44431.85 \\
\hline d1655 & 4232 & 2882 & 494 & 1235 & 2.344 .71 & 0.031 & 43 & 17616 & 92.42 & 3462.87 & 13426.57 & 16981.86 \\
\hline vm1748 & 3301 & 2412 & 709 & 1353 & 2.292 .96 & 0.000 & 1 & 29 & 105.07 & 381.21 & 1.43 & 487.71 \\
\hline $\mathrm{u} 1817$ & 11066 & 5393 & 61 & 1379 & 2.323 .68 & 0.003 & 3 & 12853 & 12711.96 & 68964.49 & 15653.42 & 97329.87 \\
\hline rl1889 & 2847 & 2415 & 1005 & 1598 & $2.18 \quad 1.71$ & 0.000 & 1 & 9 & 33.54 & 130.51 & 0.63 & 164.68 \\
\hline d2103 & 7312 & 5381 & 66 & 1985 & 2.060 .95 & 0.000 & 1 & 8 & 326.33 & 6170.10 & 1.45 & 6497.88 \\
\hline $\mathrm{u} 2152$ & 12094 & 5861 & 212 & 1578 & 2.364 .23 & 0.000 & 1 & 324 & 36195.36 & 66704.89 & 26.97 & 102927.22 \\
\hline u2319 & - & - & & - & - & - & - & - & - & - & - & - \\
\hline pr2392 & 6313 & 3676 & 521 & 1519 & 2.574 .99 & 0.000 & 1 & 23 & 189.22 & 742.03 & 2.56 & 933.81 \\
\hline pcb3038 & 12553 & 5529 & 470 & - & - & - & - & 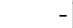 & 48677.24 & 44133.20 & - & - \\
\hline fl3795 & - & - & & - & - & - & - & - & - & - & - & - \\
\hline fnl4461 & 17124 & 8029 & 389 & - & - & - & - & - & 17103.32 & 12753.33 & - & - \\
\hline rl5915 & 10090 & 8679 & 3418 & 5337 & $2.11 \quad 1.42$ & 0.000 & 1 & 29 & 620.76 & 1412.03 & 5.38 & 2038.17 \\
\hline rl5934 & 10154 & 8767 & 3083 & 5256 & $2.13 \quad 1.37$ & 0.000 & 1 & 15 & 709.07 & 2469.14 & 5.13 & 3183.34 \\
\hline pla7397 & - & - & & - & - & 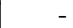 & - & 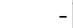 & - & - & - & - \\
\hline rl11849 & - & - & & - & - & - & - & - & - & - & - & - \\
\hline usa13509 & - & - & - & - & - & - & - & - & - & - & - & - \\
\hline brd14051 & - & - & - & - & - & - & - & - & - & - & - & - \\
\hline d15112 & - & - & - & - & - & - & - & - & - & - & - & - \\
\hline d18512 & - & - & & - & - & - & - & - & - & - & - & - \\
\hline pla33810 & - & - & & - & - & - & - & 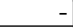 & - & - & - & - \\
\hline
\end{tabular}

Table 12. Hexagonal metric. TSPLIB instances. 


\begin{tabular}{|c|c|c|c|c|c|c|c|c|c|c|c|c|}
\hline \multirow[b]{2}{*}{ Instance } & \multicolumn{3}{|c|}{ FST counts } & \multicolumn{2}{|c|}{ SMT properties } & \multicolumn{3}{|c|}{ FST conc } & \multicolumn{4}{|c|}{ CPU time } \\
\hline & Gen & Prun & Req & NumF & SizeF Red & Gap & Nodes & LPs & Gen & Prun & Conc & Total \\
\hline d198 & 277 & 239 & 90 & 153 & 2.291 .67 & 0.000 & 1 & 4 & 0.28 & 12.78 & 0.02 & 13.08 \\
\hline $\operatorname{lin} 318$ & 1320 & 532 & 35 & 210 & 2.515 .83 & 0.000 & 1 & 73 & 7.82 & 152.74 & 0.80 & 161.36 \\
\hline fl 417 & 1357 & 653 & 125 & 217 & $2.92 \quad 2.97$ & 0.000 & 1 & 8 & 6.69 & 256.11 & 0.22 & 263.02 \\
\hline pcb442 & 680 & 529 & 228 & 318 & 2.392 .88 & 0.035 & 5 & 33 & 1.26 & 31.88 & 0.69 & 33.83 \\
\hline att532 & 1706 & 496 & 158 & 313 & 2.704 .68 & 0.000 & 1 & 11 & 21.43 & 258.74 & 0.09 & 280.26 \\
\hline ali535 & 1390 & 493 & 228 & 359 & 2.494 .05 & 0.000 & 1 & 209 & 16.61 & 288.51 & 1.74 & 306.86 \\
\hline $\mathrm{u} 574$ & 1298 & 536 & 237 & 377 & 2.523 .82 & 0.000 & 1 & 7 & 10.96 & 140.89 & 0.16 & 152.01 \\
\hline rat575 & 1784 & 670 & 117 & 343 & $2.67 \quad 4.97$ & 0.000 & 1 & 15 & 28.73 & 79.14 & 1.14 & 109.01 \\
\hline p654 & 1466 & 1014 & 136 & 328 & 2.993 .41 & 0.050 & 419 & 2685 & 5.29 & 222.32 & 3204.50 & 3432.11 \\
\hline d657 & 1575 & 660 & 254 & 431 & $2.52 \quad 3.92$ & 0.000 & 1 & 11 & 17.78 & 454.49 & 0.11 & 472.38 \\
\hline $\operatorname{gr666}$ & 2138 & 626 & 207 & 382 & $2.74 \quad 4.62$ & 0.000 & 1 & 18 & 34.35 & 734.36 & 0.19 & 768.90 \\
\hline $\mathrm{u} 724$ & 1389 & 723 & 307 & 513 & 2.413 .48 & 0.000 & 1 & 6 & 12.63 & 47.23 & 0.17 & 60.03 \\
\hline $\operatorname{rat} 783$ & 2331 & 843 & 178 & 491 & 2.594 .98 & 0.000 & 1 & 6 & 44.93 & 146.73 & 0.12 & 191.78 \\
\hline dsj1000 & 2887 & 824 & 394 & 583 & 2.714 .16 & 0.000 & 1 & 5 & 97.30 & 1247.34 & 0.14 & 1344.78 \\
\hline pr1002 & 1967 & 998 & 451 & 688 & $2.45 \quad 3.40$ & 0.000 & 1 & 4 & 20.83 & 375.04 & 0.18 & 396.05 \\
\hline u1060 & 3179 & 1254 & 466 & 693 & 2.533 .26 & 0.000 & 1 & 1292 & 50.00 & 1786.55 & 91427.58 & 93264.13 \\
\hline $\mathrm{vm} 1084$ & 1933 & 1045 & 674 & 838 & 2.293 .41 & 0.000 & 1 & 12 & 28.25 & 85.37 & 0.27 & 113.89 \\
\hline pcb1173 & 2586 & 1119 & 551 & 798 & 2.473 .15 & 0.000 & 1 & 7 & 51.11 & 740.75 & 0.17 & 792.03 \\
\hline d1291 & 2776 & 2097 & 233 & - & - & - & - & 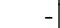 & 18.07 & 1282.39 & - & - \\
\hline rl1304 & 1869 & 1339 & 906 & 1123 & 2.161 .93 & 0.000 & 1 & 6 & 16.91 & 151.40 & 0.16 & 168.47 \\
\hline rl1323 & 1775 & 1324 & 948 & 1151 & $2.15 \quad 1.83$ & 0.000 & 1 & 4 & 17.82 & 159.96 & 0.15 & 177.93 \\
\hline nrw1379 & 5209 & 1577 & 266 & 786 & 2.755 .52 & 0.000 & 1 & 59 & 289.63 & 688.16 & 4.67 & 982.46 \\
\hline fl 1400 & 16860 & 6697 & 184 & 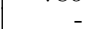 & - & - & - & 0 & 752.03 & 34119.33 & - & 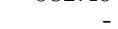 \\
\hline u1432 & 2402 & 2029 & 342 & 863 & $2.66 \quad 3.34$ & 0.043 & 6618 & 8178 & 8.55 & 58.83 & 1423.10 & 1490.48 \\
\hline fl1577 & 4356 & 2635 & 494 & 1209 & 2.301 .62 & 0.000 & 1 & 102 & 71.09 & 3847.04 & 6.15 & 3924.28 \\
\hline d1655 & 2749 & 2195 & 664 & 1244 & 2.332 .45 & 0.019 & 83 & 1403 & 23.49 & 1600.79 & 873.80 & 2498.08 \\
\hline $\mathrm{vm} 1748$ & 3306 & 1695 & 1019 & 1335 & 2.313 .51 & 0.000 & 1 & 8 & 92.25 & 264.12 & 0.79 & 357.16 \\
\hline $\mathrm{u} 1817$ & 3715 & 2938 & 161 & - & - & - & - & 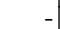 & 38.69 & 680.79 & - & - \\
\hline rl1889 & 2789 & 1892 & 1303 & 1610 & $2.17 \quad 2.29$ & 0.000 & 1 & 8 & 48.84 & 232.01 & 0.43 & 281.28 \\
\hline d2103 & 4447 & 3474 & 177 & 1919 & 2.100 .83 & 0.000 & 1 & 13 & 55.68 & 3794.06 & 1.48 & 3851.22 \\
\hline $\mathrm{u} 2152$ & 3971 & 3375 & 260 & 1548 & 2.392 .22 & 0.000 & 1 & 166 & 43.68 & 179.66 & 22.80 & 246.14 \\
\hline $\mathrm{u} 2319$ & 5975 & 4176 & 92 & - & - & - & - & - & 34.58 & 190.78 & - & - \\
\hline pr2392 & 4866 & 2654 & 1134 & 1688 & $2.42 \quad 3.69$ & 0.000 & 1 & 9 & 119.54 & 240.68 & 1.30 & 361.52 \\
\hline pcb3038 & 10898 & 4015 & 844 & 1900 & 2.603 .57 & 0.003 & 19 & 151 & 1004.02 & 12879.92 & 46.90 & 13930.84 \\
\hline fl3795 & & & & & - & & - & & & & & - \\
\hline fnl 4461 & 17014 & 5184 & 727 & 2464 & 2.815 .45 & 0.000 & 1 & 458 & 7400.69 & 4035.67 & 556.04 & 11992.40 \\
\hline rl5915 & 9400 & 6924 & 3798 & 5224 & $2.13 \quad 1.95$ & 0.000 & 1 & 23 & 635.37 & 1618.47 & 5.19 & 2259.03 \\
\hline rl5934 & 9902 & 7043 & 3439 & 5172 & $2.15 \quad 1.80$ & 0.000 & 1 & 177 & 858.25 & 3597.12 & 35.64 & 4491.01 \\
\hline pla7397 & 17429 & 11966 & 1766 & & - & - & - & - & 2456.84 & 55923.99 & 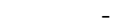 & - \\
\hline rl11849 & 21470 & 14421 & 6298 & 9468 & 2.252 .30 & 0.000 & 3 & 137 & 6010.50 & 23773.68 & 257.75 & 30041.93 \\
\hline usa13509 & 39254 & 12801 & 4533 & - & - & - & - & 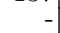 & 41525.14 & 56671.60 & - & - \\
\hline brd14051 & - & - & - & - & - & - & - & - & - & - & - & - \\
\hline $\mathrm{d} 15112$ & - & - & - & - & - & - & - & - & - & - & - & - \\
\hline d18512 & - & - & - & - & - & - & - & 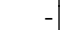 & - & - & - & - \\
\hline pla33810 & - & - & - & - & - & - & - & - & - & - & - & - \\
\hline
\end{tabular}

Table 13. Octilinear metric. TSPLIB instances. 